\title{
Characterisations of Pseudo-Amenability
}

by

Aleksa Vujičić

A thesis

submitted to the Victoria University of Wellington

in fulfilment of the

requirements for the degree of

Master of Science

in Mathematics.

Victoria University of Wellington

2019 



\begin{abstract}
We start this thesis by introducing the theory of locally compact groups and their associated Haar measures. We provide examples and prove important results about locally compact and more specifically amenable groups. One such result is known as the Følner condition, which characterises the class amenable groups. We then use this characterisation to define the notion of a pseudo-amenable group. Our central theorem that we present provides new characterisations of pseudo-amenable groups. These characterisations allows us to prove several new results about these groups, which closely mimic well known results about amenable groups. For instance, we show that pseudo-amenability is preserved under closed subgroups and homomorphisms.
\end{abstract}




\section{Acknowledgments}

I would like to thank my supervisor Hung Le Pham for his guidance and support throughout the duration of my thesis. A big thank you to my parents as well for their love, patience, and proof-reading skills.

Thank you also to all the people in my office, whom I've shared many interesting conversations and experiences. 


\section{Contents}

$\begin{array}{lll}1 & \text { Introduction } & 1\end{array}$

1.1 History $\ldots \ldots \ldots \ldots \ldots \ldots \ldots$

1.2 Overview . . . . . . . . . . . . . . . . . . . 2

1.3 Background . . . . . . . . . . . . . . . 4

2 The Haar Measure 5

$2.1 \quad$ Locally Compact Spaces . . . . . . . . . . . . . . . . . . 5

2.2 Topological Groups . . . . . . . . . . . . . . . . . . 11

2.3 Existence \& Uniqueness . . . . . . . . . . . . . . . . . . . 13

2.4 Properties of the Haar Measure . . . . . . . . . . . . . . 24

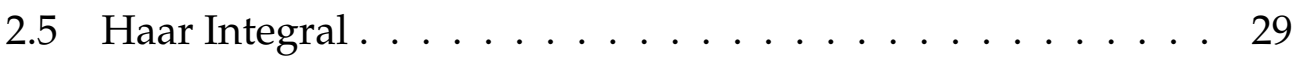

$\begin{array}{lll}3 & \text { Amenability } & 39\end{array}$

$3.1 \quad$ Paradoxical Decompositions. . . . . . . . . . . . . . . . . . 39

3.2 Left-Invariant Means . . . . . . . . . . . . . . . . . . . . 43

3.3 Følner Conditions . . . . . . . . . . . . . . . . . . . . . 46

4 Pseudo-Amenability 55

4.1 Definition . . . . . . . . . . . . . . . . . . . 55

4.2 Characterisations . . . . . . . . . . . . . . . . 60

4.3 Subgroups \& Homomorphisms . . . . . . . . . . . . . 68

4.4 Similarities to Amenability . . . . . . . . . . . . . . . 77 


\section{Chapter 1}

\section{Introduction}

\subsection{History}

The theory of amenability began with measure theory, which was famously introduced by Lebesgue in the early twentieth century.

The Haar measure was introduced by Alfréd Haar in 1933 [9]. This was important as it allowed for the construction of a measure which respected the group structure. This meant that it would be possible to incorporate many aspects of measure theory and functional analysis into the study of (locally compact) groups. Haar proved in his paper the existence and uniqueness of such a measure, and we shall closely follow his proof in this thesis.

The notion of amenability was first introduced by Von Neumann as a response to the Banach-Tarski Paradox. Informally, this paradox tells us that we can partition the sphere in three dimensions and translate the individual components in such a way so that we obtain two copies of our original sphere. Notably, this paradox was proven to only exist in dimensions three or greater. This was shown to be a property of the underlying group of rigid motions, and such groups where this did not occur were shown to possess an invariant finitely-additive measure. This was used by Von Neumann to define the class of amenable groups. 
Later, in the 1950s, Day shifted the focus from finitely-additive measures to left-invariant means [3]. Day also introduced the term 'amenable group' here as a pun. This shift to invariant means was also important as it now makes it possible to use the machinery of functional analysis in the study of amenability. As a result, the subject of amenable groups has received much activity in the past several decades. Amenable groups have applications in many other disciplines of mathematics as well, such as Lie groups and Ergodic theory.

\subsection{Overview}

A major focus of this thesis will be to introduce the notion of pseudoamenability in locally compact groups, and explore some properties of these groups. Before we can do this however, we shall review the existing theory that is necessary to work with such groups.

In Chapter 2 we present the definition of a locally compact group, and prove the existence and uniqueness of a Haar measure on this group. A Haar measure is a Borel regular measure that is finite on compact sets, and is invariant under left translations. We shall use this Haar measure to explore some of the structure on the group, and more importantly use it to define some important spaces of functions. Of particular interest will be the Lebesgue spaces $L^{p}(G)$, and the space of Borel regular measures $M(G)$. This chapter will follow the material presented in Cohn [1] and Loomis [13].

We shall then use the Haar measure in Chapter 3 to define and study the notion of amenability. We motivate this by introducing the BanachTarski paradox, and discussing paradoxical decompositions on discrete groups. This will lead us to the following definition of amenability.

Definition. We say that a locally compact group $G$ is amenable if it has a leftinvariant mean (on $L^{\infty}(G)$ ). 
We shall define the notion of a mean precisely, but it can be in essence seen as a sort of integral. We will then show several equivalent formulations of amenability, one of these being the Følner condition, given below.

(FC) For every $\varepsilon>0$ and compact $K \subseteq G$, there is some Borel set $U$ so that $0<\lambda(U)<\infty$ and

$$
\lambda(x U \Delta U)<\varepsilon \lambda(U)
$$

for every $x \in K$.

We shall primarily follow the books of Greenleaf [8] and Paterson [18].

Finally, we introduce and study the properties of pseudo-amenability in Chapter 4. We present two new characterisations of pseudo-amenability in the form of the following conditions.

(L) For every $\varepsilon \in(0,1)$, there is some $N \in \mathbb{N}$ such that for all $n \geq N$, and all $s_{1}, \ldots, s_{n} \in G$ (not necessarily distinct), there is some $f \in L^{1}(G)^{+}$ so that

$$
\left\|s_{1} * f \vee \ldots \vee s_{n} * f\right\|<\varepsilon n\|f\|
$$

(M) For every $\varepsilon \in(0,1)$, there is some $N \in \mathbb{N}$ such that for all $n \geq N$, and all $s_{1}, \ldots, s_{n} \in G$ (not necessarily distinct), there is some $\mu \in M(G)^{+}$ so that

$$
\left\|s_{1} * \mu \vee \ldots \vee s_{n} * \mu\right\|<\varepsilon n\|\mu\|
$$

The central theorem of this section is the following.

Theorem. Let $G$ be a locally compact group. Then the following are equivalent:

- $G$ is pseudo-amenable.

- G satisfies (L).

- G satisfies (M).

This result will allow us to characterise some of the basic properties of pseudo-amenable groups, such as pseudo-amenability being preserved under subgroups. 


\subsection{Background}

We shall assume that the reader has some familiarity with the relevant background material. In particular, we assume working knowledge of measure theory and functional analysis. We shall review some of the more specific concepts as we need them, however we assume the reader is familiar with basic definitions and results (such as the monotone convergence theorem in measure theory). We shall also make use of some elementary group theory and topology. 


\section{Chapter 2}

\section{The Haar Measure}

\subsection{Locally Compact Spaces}

The ultimate aim of this chapter will be to construct a Haar measure. We shall define this term precisely later; however in essence, this is a 'wellbehaved' measure on a group which respects the group structure in a certain sense. This Haar measure will be particularly useful in studying the analytic properties of the spaces on which they arise. For instance, one can use this measure to define an integral, which in turn can be used to define the Lebesgue spaces of functions ( $L^{p}$ spaces) on this space.

Haar measures arise on structures known as locally compact groups these are groups which are equipped with a locally compact topological structure that is compatible with the group operations.

In this chapter, we follow closely the definitions and proofs presented in Cohn [1, Chapters 7 \& 9] and Loomis [13, Chapter 6]. We start by introducing the type of topological structures we are interested in, as well as a few results that we shall later use.

Definition 2.1.1. A topological space $X$ is Hausdorff if for any distinct $x, y \in$ $X$, there are disjoint open sets $U$ and $V$ so that $x \in U$ and $y \in V$.

Many 'nice' topologies have the property of being Hausdorff. It is a 
condition that will give rise to many other nice results about the space. For instance, in a Hausdorff space, one can show that all limits are unique or that all compact sets are closed (in fact, one could derive the latter as a corollary of Proposition 2.1.2.

By definition, being Hausdorff essentially allows us to separate any two points using open sets. However, we can generalise this fact - we can separate any two compact sets using open sets as follows.

Proposition 2.1.2. Let $X$ be a Hausdorff space, and $K$ and $L$ disjoint compact subsets of $X$. Then there exist disjoint open subsets $U$ and $V$ containing $K$ and $L$ respectively.

Proof. First we consider the case where $K$ is the singleton $\{x\}$. Since $X$ is Hausdorff, then for every $y \in L$ there are open sets $U_{y}, V_{y}$ so that $x \in$ $U_{y}, y \in V_{y}$, and $U_{y} \cap V_{y}=\emptyset$. Now, $\left\{V_{y}\right\}_{y \in L}$ is an open cover of $L$. By compactness of $L$, there are $y_{1}, \ldots, y_{n} \in L$ so that $L \subseteq \bigcup_{i=1}^{n} V_{y_{i}}$. If we define $U=\bigcap_{i=1}^{n} U_{y_{i}}$ and $V=\bigcup_{i=1}^{n} V_{y_{i}}$, then these are precisely the open sets we require.

For the general case, we have that $K$ is some arbitrary compact set. However, for every $x \in K$, we know by the previous argument that there are some open sets $U_{x}$ and $V_{x}$ so that $x \in U_{x}, L \subseteq V_{x}$ and $U_{x} \cap V_{x}=\emptyset$. Now, we have that $\left\{U_{x}\right\}_{x \in K}$ is an open cover of $K$, so by compactness there are $x_{1}, \ldots, x_{n} \in K$ so that $K \subseteq \bigcup_{i=1}^{n} U_{x_{i}}$. Taking $U=\bigcup_{i=1}^{n} U_{x_{i}}$ and $V=\bigcap_{i=1}^{n} V_{x_{i}}$, we obtain the required open sets.

We can then use this result to split compact sets in the following way.

Lemma 2.1.3. Let $X$ be Hausdorff and $K$ a compact set. Let $U_{1}, U_{2}$ be open sets so that $K \subseteq U_{1} \cup U_{2}$. Then we can find compact sets $K_{1}, K_{2}$ so that $K_{1} \subseteq U_{1}$, $K_{2} \subseteq U_{2}$, and $K=K_{1} \cup K_{2}$.

Proof. Let $L_{1}=K \backslash U_{1}$ and $L_{2}=K \backslash U_{2}$. These are both compact as a closed subset of a compact set is compact. We can invoke Proposition 2.1.2 so that 
we can find disjoint open sets $V_{1}$ and $V_{2}$, so that $L_{1} \subseteq V_{1}$ and $L_{2} \subseteq V_{2}$. Now let $K_{1}=K \backslash V_{1}$ and $K_{2}=K \backslash V_{2}$, which are also compact.

To show that these are our required sets, we need to show that $K_{1} \subseteq U_{1}$, $K_{2} \subseteq U_{2}$, and $K=K_{1} \cup K_{2}$. We can see the latter is true as

$$
K_{1} \cup K_{2}=K \backslash\left(V_{1} \cap V_{2}\right)=K
$$

Furthermore, we can verify that $K_{1} \subseteq U_{1}$ as

$$
K_{1} \backslash U_{1}=\left(K \backslash V_{1}\right) \backslash U_{1}=\left(K \backslash U_{1}\right) \backslash V_{1}=L_{1} \backslash V_{1}=\emptyset
$$

Likewise, we have that $K_{2} \subseteq U_{2}$, thus proving our claim.

Naturally, one can extend this proposition for any finite number of open sets.

Corollary 2.1.4. Let $U=\bigcup_{i=1}^{n} U_{i}$ where each $U_{i}$ is open. Let $K$ be a compact subset of $U$. Then there are compact sets $K_{i}$, so that $K_{i} \subseteq U_{i}$ for every $i=1 \ldots n$, and that $K=\bigcup_{i=1}^{n} K_{i}$.

As a reminder, our aim is to construct a measure that is invariant to translations. As we shall see, the construction of such a measure is done using compact sets. However, for this process to successfully give us the measure we desire, we need every point to be contained in a 'sufficiently large' compact set. For this, we introduce the notion of a compact neighbourhood and present the following definition.

Definition 2.1.5. A Hausdorff space $X$ is called locally compact if for any point $x \in X$, there is a neighbourhood $U$ of $x$ and a compact set $K$ so that

$$
x \in U \subseteq K
$$

We say that $K$ is a compact neighbourhood of $x$.

In our definition, we require $X$ to be Hausdorff in order to be locally compact. Some authors will work with non-Hausdorff locally compact 
spaces. However, for our concerns, we will not need to look at these spaces. As such, we shall assume that any locally compact space is automatically Hausdorff.

We note the definition of locally compact is equivalent to saying that about any point $x$, we can find a neighbourhood $U$ of $x$ with compact closure. We can see this as if we have $x \in U \subseteq K$, with $U$ open and $K$ compact, then $\bar{U}$ is a closed subset of $K$, and thus compact. The other direction for this equivalence is trivial.

Local compactness allows us to obtain a few even stronger topological properties. To start with we can show that all compact sets (not just the singletons) are contained in an open set with compact closure.

Lemma 2.1.6. Let $X$ be a locally compact space. Let $K$ be a compact set. Then there is an open set $U$ so that $K \subseteq U$ and $U$ has compact closure.

Proof. For every $x \in K$, let $U_{x}$ be an open set containing $x$ with compact closure. This gives us then that the collection of $U_{x}$ forms an open cover of $K$. As $K$ is compact, we can find a finite subcover $\left\{U_{i}: i=1, \ldots, n\right\}$. If we let $U=\bigcup_{i=1}^{n} U_{i}$, we have that $K \subseteq U$, and furthermore we can see that $U$ has compact closure as each $U_{i}$ does as well.

We can improve this result. We can extend it to say that the open set we find (with compact closure) can be in a certain sense as small as we like.

Proposition 2.1.7. Let $X$ be a locally compact space. Let $K$ be a compact set and $U$ an open set containing $K$. Then there is an open set $V$ with compact closure so that

$$
K \subseteq V \subseteq \bar{V} \subseteq U
$$

Proof. Let $W$ be an open set containing $K$ with compact closure. We can replace $W$ by $W \cap U$, so we can assume without loss of generality that $W \subseteq$ $U$. This $W$ almost works as our candidate, but unfortunately it is possible that $\bar{W}$ extends outside of $U$. To mitigate this, we let $L=\bar{W}-W$ (which 
is compact), and use Proposition 2.1.2 on $L$ and $K$, so that we obtain two disjoint open sets $V_{1}$ and $V_{2}$ with $K \subseteq V_{1}$ and $L \subseteq V_{2}$.

If we now set $V=V_{1} \cap W$, we can see that $V$ is our desired set. Firstly, we can see that $K \subseteq V$. Next, we have that $\bar{V}$ is compact, as it is a closed subset of a compact set $(\bar{W})$. Lastly, we need to check that $\bar{V} \subseteq U$. So take $y \in \bar{V}$. This gives us that $y \in \overline{V_{1}}$ and $y \in \bar{W}$. Since $V_{1}$ and $V_{2}$ are open and disjoint, the former containment gives us that $y \notin V_{2}$. This tells us that $y \notin L$, so either $y \notin \bar{W}$ or $y \in W$. From before we have that $y \in \bar{W}$, hence we have that $y \in W \subseteq U$, and so $\overline{V_{1}} \subseteq U$.

We also need to introduce an important space of functions, namely the continuous functions with compact support. We begin by defining the support of a function as follows.

Definition 2.1.8. Let $X$ be a topological space, and let $f$ be a function mapping $X$ to $\mathbb{C}$. Then the support of $f$ is the set

$$
\operatorname{supp}(f):=\overline{\{x \in X: f(x) \neq 0\}}
$$

We can think of the support of a function being the set of points where the function is 'interesting'. Everywhere else the function is simply 0. If, for example, two functions have disjoint supports, then one could say they act on two different parts of the space. We can use this to define the space of continuous functions with compact support.

Definition 2.1.9. Let $X$ be a topological space. We let $C_{c}(X)$ denote the set of all continuous functions from $X$ to $\mathbb{C}$ whose support is compact

A function $f$ with compact support means that it only acts on a compact space, and is zero elsewhere. This is a strong definition, and at times we need something slightly weaker. We can define another closely related space of functions.

Definition 2.1.10. Let $X$ be a topological space. A function $f: X \rightarrow \mathbb{C}$ is said to vanish at infinity if for every $\varepsilon>0$, the set $\{x \in X:|f(x)| \geq \varepsilon\}$ is contained 
in a compact set. We let $C_{0}(X)$ denote the set of all continuous functions that vanish at infinity.

It is clear from the definition that functions with compact support will always vanish at infinity.

We can use these functions to separate sets in a certain sense. To do this we will need a result known as Urysohn's Lemma, which we state here without proof.

Theorem 2.1.11 (Urysohn's Lemma). Let $X$ be a normal topological space, and let $E, F \subseteq X$ be disjoint closed sets. We can then find a continuous function $f: X \rightarrow[0,1]$ so that $f(x)=0$ for every $x \in e$ and $f(x)=1$ for every $x \in F$.

Here, a topological space $X$ is normal if every pair of disjoint closed subsets of $X$ can be separated by a pair of disjoint open subsets. We can see by Proposition 2.1.2 that any compact Hausdorff set is normal. Unfortunately we will be working with spaces which are not necessarily normal, so we cannot use Urysohn's Lemma directly.

Recall that for any Borel set $A \subseteq X$, we can define a characteristic function by

$$
\chi_{A}(x):= \begin{cases}1 & x \in A \\ 0 & x \notin A\end{cases}
$$

Using this, we have the following weaker version of Urysohn's Lemma for locally compact spaces.

Proposition 2.1.12. Let $X$ be a locally compact set. Let $K$ be a compact set and $U$ an open set so that $K \subseteq U$. Then there exists a function $f \in C_{c}(X)$ so that $\chi_{K} \leq f \leq \chi_{U}$.

Proof. We can use Proposition 2.1.7 to find an open set $V$ with compact closure so that $K \subseteq V \subseteq \bar{V} \subseteq U$. Now $\bar{V}$ is a compact Hausdorff space and is thus normal. By Urysohn's Lemma, we can find a continuous function $g: \bar{V} \rightarrow[0,1]$ so that $g(x)=1$ for $x \in K$ and $g(x)=0$ for $x \in \bar{V} \backslash V$. 
Now define $f: X \rightarrow[0,1]$ by

$$
f(x)= \begin{cases}g(x) & x \in \bar{V} \\ 0 & \text { otherwise }\end{cases}
$$

It is clear from the definition that $\chi_{K} \leq f \leq \chi_{U}$. We can check that $f$ is continuous as it is continuous on the closed sets $\bar{V}$ and $X \backslash V$. Moreover we have that $\operatorname{supp}(f) \subseteq \bar{V}$, so $\operatorname{supp}(f)$ must be compact, and therefore $f \in C_{c}(X)$.

\subsection{Topological Groups}

We now wish to extend our topological space by adding a group structure. This group structure should be compatible with the topology, and we can do this as follows.

Definition 2.2.1. Let $G$ be a group with a topology on it. We call $G$ a topological group if the group operations $\left((a, b) \mapsto a \cdot b\right.$ and $\left.a \mapsto a^{-1}\right)$ are continuous with respect to this topology.

When dealing with general topological spaces, we often deal with subsets of our space, rather than individual elements. With this in mind, we need a way of applying the group operation on subsets of our group. Recall that for any arbitrary function $f: X \rightarrow Y$, we can implicitly define $f(A)=\{f(x): x \in A\}$ for any $A \subseteq X$. We extend this notion to the group operation and group inverses, so that for any $x \in G$ and $A \subseteq G$, we have

$$
\begin{aligned}
x A & :=\{x y: y \in A\} \\
A^{-1} & :=\left\{y^{-1}: y \in A\right\}
\end{aligned}
$$

As the group operation and inverse are continuous, it should be no surprise that this operation preserves the topological structure.

Proposition 2.2.2. Let $U$ be an open/closed/compact set. Then for any $a \in G$, the sets $a U$ and $U^{-1}$ are also open/closed/compact respectively. 
Proof. This follows as the mapping $x \mapsto a x$ is a homeomorphism. This can be seen as it is bijective and continuous, and its inverse $\left(x \mapsto a^{-1} x\right)$ is also continuous. Likewise the mapping $x \mapsto x^{-1}$ is also a homeomorphism.

This proposition will prove to be incredibly useful, as it means that any topological group must have a topology which is invariant under both translations and inverses. And indeed, this will be instrumental in constructing a left-invariant measure.

For now, we proceed with the following propositions. We note that we say that a set $A$ is symmetric if $A=A^{-1}$.

Proposition 2.2.3. Let $U$ be an open neighbourhood of the identity e. Then there is an (open) neighbourhood $V$ of e so that $V V$ is contained in $U$. Moreover we can also find a symmetric neighbourhood of e that is also contained in $U$.

Proof. We know the group operation is continuous. So then the set $W=$ $\{(x, y) \in G \times G: x y \in U\}$ is open in the product topology (as $W$ is the preimage of $U)$. Since $(e, e) \in W$, it follows by the definition of a product topology, that there are some neighbourhoods $V_{1}, V_{2} \subseteq G$ of $e$ so that $V_{1} \times$ $V_{2} \subseteq W$. Let $V=V_{1} \cap V_{2}$. We can see now that $V V \subseteq V_{1} V_{2} \subseteq U$, thus proving the first part of the proposition.

For the second part, we note that both $e \in U$ and $e \in U^{-1}$. By continuity of the group inverse, we know that $U^{-1}$ is open, so that gives us that $U \cap$ $U^{-1}$ is a symmetric neighbourhood of $e$ contained in $U$.

Proposition 2.2.4. Let $G$ be a topological group, and $H$ a subgroup of $G$. If $H$ is open, then $H$ is also closed.

Proof. Since $H$ is open, we know all of its cosets are open as well (as they are simply translations of $H$ ). The complement of $H$ is simply the union of all its other cosets, so must be open. Hence $H$ is closed.

Proposition 2.2.5. Let $G$ be a topological group. Let $K \subseteq G$ be compact, and let $U$ be an open set so that $K \subseteq U$. Then there are open neighbourhoods $V_{L}$ and $V_{R}$ of the identity e, so that $V_{L} K \subseteq U$ and $K V_{R} \subseteq U$. 
Proof. First take any $x \in K$. Then, we know (by Proposition 2.2.2) that $x^{-1} U$ is an open neighbourhood of $e$. So we can use Proposition 2.2.3 to find an open neighbourhood $V_{x}$ of $e$ so that $V_{x} V_{x} \subseteq x^{-1} U$. Now, $\left\{x V_{x}\right\}_{x \in K}$ is an open cover of $K$. So take finitely many $x_{i}$ so that $x_{i} V_{x_{i}}$ for $i=1, \ldots, n$ cover $K$. Now define $V_{R}=\bigcap_{i=1}^{n} V_{x_{i}}$. So for any $y \in K$, there is some $x_{i}$ so that $y \in x_{i} V_{x_{i}}$. Hence we have that

$$
y V_{R} \subseteq y V_{x_{i}} \subseteq x_{i} V_{x_{i}} V_{x_{i}} \subseteq x_{i} x_{i}^{-1} U=U
$$

Since this holds for all $y \in K$, then we have that $K V_{R} \subseteq U$. A similar argument may be used to construct $V_{L}$.

Naturally, we can also define locally compact groups in the obvious way.

Definition 2.2.6. A topological group $G$ is locally compact if its topology is locally compact.

\subsection{Existence \& Uniqueness}

We finally have the background to introduce the Haar measure. This measure will aid us in our study of amenable (and pseudo-amenable) locally compact groups. We explore this connection in more depth in Chapters 3 and 4

First however, we need to define precisely what a Haar measure is. We begin by defining the following types of measures.

Definition 2.3.1. Let $\mu$ be a non-trivial countably additive measure on the Borel sets of a topological space $X$. Then $\mu$ is a Borel regular measure (or simply regular measure) if it satisfies the following:

- $\mu$ is outer regular on Borel sets:

$$
\mu(S)=\inf \{\mu(U): U \text { is open and } S \subseteq U\}
$$

for every Borel set $S \subseteq X$ 
- $\mu$ is inner regular on open sets:

$$
\mu(U)=\sup \{\mu(C): C \text { is compact and } C \subseteq U\}
$$

for every open set $U \subseteq X$

Definition 2.3.2. Let $\mu$ be a (Borel) regular measure on a topological space $X$. We say $\mu$ is a Radon measure if it is locally finite. That is for every $x \in X$, there is an open neighbourhood $U$ of $x$ so that $U$ is $\mu$-finite.

We note here that when dealing with locally compact spaces, the definition of a Radon measure has the following equivalent formulation.

Proposition 2.3.3. Let $\mu$ be a regular measure on a locally compact space $X$. Then $\mu$ is a Radon measure if and only if it is finite on all compact sets.

Proof. First suppose $\mu$ is a Radon measure, and let $C$ be a compact set. For every $x \in C$, let $U_{x}$ be a neighbourhood of $x$ which is $\mu$-finite. Then $\left\{U_{x}\right\}_{x \in C}$ is an open cover of $C$, so it has a finite subcover. It follows then that for some $x_{1}, \ldots, x_{n} \in C$, we have that

$$
\mu(C) \leq \mu\left(\bigcup_{i=1}^{n} U_{x_{i}}\right)=\sum_{i=1}^{n} \mu\left(U_{x_{i}}\right)<\infty
$$

In the other direction, suppose $\mu$ is finite on all compact sets. Take any $x \in X$. Then by local compactness, there is an open set $U$ and compact set $C$ so that $x \in U \subseteq C$. But since $\mu(C)$ is finite, it must follow that $\mu(U)$ is also finite.

Since we study exclusively locally compact groups, we shall regularly use this characterisation of Radon measures.

The measures we have defined so far only invoke topological properties of the underlying set. If we now suppose our underlying set is a (topological) group, we can define the notion of a translation invariant measure. 
Definition 2.3.4. Let $\mu$ be a Radon measure on a topological group G. Then $\mu$ is a (left) Haar measure if it is left-invariant. That is, for all $g \in G$ and Borel $S \subseteq G$, we have $\mu(g S)=\mu(S)$.

Finding such a measure would prove incredibly useful in analysing properties of groups. Even further, we can use this measure to define a left-invariant Haar integral and to look at elements of Lebesgue spaces of $G$. Indeed, there are several common examples of Haar measures. One of the most notable of these is the Lebesgue measure, which plays a crucial role when studying the analytic properties of $\mathbb{R}$.

In general, there is no guarantee that such a measure exists. Fortunately, certain groups facilitate the existence and uniqueness of such a measure. In particular, all locally compact groups have a Haar measure, and this result is known as Haar's Theorem.

Theorem 2.3.5 (Haar's Theorem). Let $G$ be a locally compact group. Then there exists a Haar measure $\lambda$ on $G$. Furthermore, if $\mu$ is also a Haar measure on $G$, then there is some $c>0$ so that $\mu=c \lambda$.

There is a fair amount of work involved in proving this theorem. We shall follow the proof that is laid out by Cohn [1, Chapter 9].

We first briefly present an overview of the proof. Firstly we shall find a way of comparing the size of compact sets, by using translations of open sets as a 'measuring unit' of sorts. For instance, suppose we have an open set $U$, and compact sets $K_{1}$ and $K_{2}$. If we have that we can cover $K_{1}$ by a union of 5 translations of $U$, and we can cover $K_{2}$ by a union of 3 translations of $U$, then we will say that $K_{1}$ is roughly $5 / 3$ times larger than $K_{2}$. By taking a kind of limit of smaller and smaller open sets, we can make finer and finer estimates for this ratio. We can then fix any suitable compact set to have unit size, and then we extend to other Borel sets using the regularity properties. This will yield us our desired Haar measure.

Other proofs of this theorem do exist. Some follow a similar route, where they construct a left-invariant integral on a space of functions, and 
derive the measure from there. Others take a different approach, some without invoking the axiom of choice. We refer the reader to Loomis [13, 14] for alternative proofs of Haar's Theorem using these methods. For us this is a minor issue, and we instead focus on presenting a more intuitive proof of this result.

With that, we shall now begin the proof of Haar's Theorem.

First we need some definitions. For convenience we define $\mathcal{C}$ to be the collection of all compact subsets of $G$, and $\mathcal{U}$ to be the collection of all neighbourhoods of $e$.

Definition 2.3.6. Let $K$ be a compact subset of $G$ and $V$ a subset with non-empty interior ( $\left.V^{i n t}\right)$. The covering number of $K$ with respect to $V$ is the smallest integer $n$ so that

$$
K \subseteq \bigcup_{i=1}^{n} x_{i} V
$$

for some $x_{1} \ldots x_{n} \in G$. We denote this number $n$ by $\#(K: V)$.

This definition is well defined, as $\left(x\left(V^{i n t}\right)\right)_{x \in G}$ is an open cover of $K$, and by compactness must have a finite subcover.

We now pick a 'unit set' that we will use to measure the size of other sets. So fix some compact set $K_{0}$ with non-empty interior. This set shall remain fixed throughout the remainder of this section. We define the following ratio.

Definition 2.3.7. Let $U \in \mathcal{U}$ (that is, $U$ is a neighbourhood of e). The covering ratio is the function taking compact sets to $\mathbb{R}$ by

$$
h_{U}(K):=\frac{\#(K: U)}{\#\left(K_{0}: U\right)}
$$

This function will serve as an approximation to our outer measure. Firstly however we prove the following properties about $h_{U}$.

Proposition 2.3.8. Take any $U \in \mathcal{U}, x \in G$, and any compact sets $K, K_{1}, K_{2}$. Then it follows that 
1. $0 \leq h_{U}(K) \leq \#\left(K: K_{0}\right)$

2. $h_{U}\left(K_{0}\right)=1$

3. $h_{U}(x K)=h_{U}(K)$

4. $h_{U}\left(K_{1}\right) \leq h_{U}\left(K_{2}\right)$ if $K_{1} \subseteq K_{2}$

5. $h_{U}\left(K_{1} \cup K_{2}\right) \leq h_{U}\left(K_{1}\right)+h_{U}\left(K_{2}\right)$

6. $h_{U}\left(K_{1} \cup K_{2}\right)=h_{U}\left(K_{1}\right)+h_{U}\left(K_{2}\right)$ if $K_{1} U^{-1}$ and $K_{2} U^{-1}$ are disjoint

Proof. First we claim that $\#(K: U) \leq \#\left(K: K_{0}\right) \#\left(K_{0}: U\right)$. This can be seen if we let $\#\left(K: K_{0}\right)=n$ and $\#\left(K_{0}: U\right)=m$, then there are $x_{1}, \ldots, x_{n}, y_{1}, \ldots, y_{m} \in G$ so that

$$
K \subseteq \bigcup_{i=1}^{n} x_{i} K_{0} \quad \text { and } \quad K_{0} \subseteq \bigcup_{i=1}^{m} y_{i} U
$$

It follows then that

$$
K \subseteq \bigcup_{i=1}^{n} \bigcup_{j=1}^{m} x_{i} y_{j} U
$$

and so \# $(K: U)$ is at most $m n$. Dividing through by \#( $\left.K_{0}: U\right)$ yields us item 1 .

Items 2 to 5 can all be derived easily from the definition.

For item 6 let $n=\#\left(K_{1} \cup K_{2}: U\right)$ with $x_{1}, \ldots, x_{n} \in G$ so that we have $K_{1} \cup K_{2} \subseteq \bigcup_{i=1}^{n} x_{i} U$. Now, each $x_{i} U$ will only meet with one of $K_{1}$ or $K_{2}$, otherwise we would have $x \in K_{1} U^{-1} \cap K_{2} U^{-1}$. This means that the sequence $x_{i}$ can be partitioned into two subsequences $y_{i}$ and $z_{i}$ so that

$$
K_{1} \subseteq \bigcup_{i=1}^{m} y_{i} U \quad \text { and } \quad K_{2} \subseteq \bigcup_{i=1}^{m} z_{i} U
$$

Using this fact and item 5 , we obtain item 6

For smaller values of $U$, the function $h_{U}$ is a finer approximation of the ratio. We can use this to obtain a function $h$ by taking a sort of limit where we shrink $U$ down to $\{e\}$. We do this by constructing the following topological space, whose members are functions from $\mathcal{C}$ to $\mathbb{R}$. Namely, for 
each $K \in \mathcal{C}$, denote $I_{K}$ to be the interval $\left[0, \#\left(K: K_{0}\right)\right]$ in $\mathbb{R}$. We then let $X=\prod_{K \in \mathcal{C}} I_{K}$, and we give $X$ the product topology. Note that by Tychonoff's Theorem, we know that $X$ is compact.

From item 11 it is clear that each $h_{U}$ is an element of $X$. Now, for any $V \in \mathcal{U}$, we define $S(V) \subseteq X$ to be

$$
S(V):=\overline{\left\{h_{U}: U \in \mathcal{U}, U \subseteq V\right\}}
$$

where $\bar{A}$ denotes the closure of $A$. Note that we trivially have that $h_{V} \in$ $S(V)$. We claim that the intersection of all $S(V)$ is non-empty.

So take finitely many $V_{1}, \ldots, V_{n} \in \mathcal{U}$. It is clear that if we let $V=\bigcap_{i=1}^{n} V_{i}$, then $S(V) \subseteq \bigcap_{i=1}^{n} S\left(V_{i}\right)$. Moreover this means that $h_{V} \in \bigcap_{i=1}^{n} S\left(V_{i}\right)$, so this intersection is non-empty. So the collection of all $S(V)$ satisfies the finite intersection property, and by compactness of $X$, this means that the intersection of all such $S(V)$ is also non-empty. So we can take and fix some $h \in \bigcap_{V \in \mathcal{U}} S(V)$.

We can show the following facts about $h$, similar to those in Proposition 2.3.8.

Proposition 2.3.9. For every $K, K_{1}, K_{2} \in \mathcal{C}$, and $x \in G$, the chosen function $h$ satisfies the following:

1. $0 \leq h(K)$

2. $h(\emptyset)=0$

3. $h\left(K_{0}\right)=1$

4. $h(x K)=h(K)$

5. $h\left(K_{1}\right) \leq h\left(K_{2}\right)$ if $K_{1} \subseteq K_{2}$

6. $h\left(K_{1} \cup K_{2}\right) \leq h\left(K_{1}\right)+h\left(K_{2}\right)$

7. $h\left(K_{1} \cup K_{2}\right)=h\left(K_{1}\right)+h\left(K_{2}\right)$ if $K_{1}$ and $K_{2}$ are disjoint

Proof. It follows immediately from the definition of $X$ that item 1 will hold. For items 2 to 6 , we can prove these all in a similar manner. We first note 
that elements of $X$ are functions from $\mathcal{C}$ to $\mathbb{R}$, and moreover that for any $K \in \mathcal{C}$, the mapping from $X$ to $\mathbb{R}$ given by $F \mapsto F(K)$ is a continuous map.

Now we prove item 6 . Take any $K_{1}, K_{2} \in \mathcal{C}$. Consider the mapping

$$
F \mapsto F\left(K_{1}\right)+F\left(K_{2}\right)-F\left(K_{1} \cup K_{2}\right)
$$

which is continuous. Now, this map is non-negative at each $h_{U}$ (by item 5 in proposition 2.3.8). This means that it is non-negative on each $S(U)$, and so must be non-negative at $h$. This proves item 6 .

We can repeat this form of argument for each of the items 2 to 5 , replacing the mapping from $X$ to $\mathbb{R}$ as needed. For instance, to show item 4 holds, we can use the mapping $F \mapsto F(K)-F(x K)$ (which is zero on each $\left.h_{U}\right)$.

Lastly, we need to prove item 7. So let $K_{1}$ and $K_{2}$ be disjoint compact sets. By Proposition 2.1.2, we can separate these by two open sets, that is, there are disjoint open sets $U_{1}, U_{2}$ such that $K_{i} \subseteq U_{i}$ for $i=1,2$. From Proposition 2.2.5, we can find two open neighbourhoods $V_{1}, V_{2} \in \mathcal{U}$, so that $K_{i} V_{i} \subseteq U_{i}$. If we let $V=V_{1} \cap V_{2}$, then we know that $K_{1} V$ and $K_{2} V$ are disjoint, and moreover, since $V \in \mathcal{U}$, then by item 6 of Proposition 2.3.8. we have that

$$
h_{V^{-1}}\left(K_{1} \cup K_{2}\right)=h_{V^{-1}}\left(K_{1}\right)+h_{V^{-1}}\left(K_{2}\right)
$$

This will also hold true if we replace $V^{-1}$ by any $U \in \mathcal{U}$ such that $U \subseteq V^{-1}$. If we again consider the map as in eq. 2.1), then we see that it vanishes at each point in $S\left(V^{-1}\right)$. Since $h \in S\left(V^{-1}\right)$, item 7 follows.

Now, $h$ is only defined on the compact subsets of $G$, so we want to extend it to all subsets of $G$. We do this by defining an outer measure as follows.

Definition 2.3.10. We define $\hat{\lambda}$ by first extending to the open sets as follows

$$
\hat{\lambda}(U)=\sup \{h(K): K \in \mathcal{C}, K \subseteq U\}
$$

and then further extending it to all subsets by

$$
\hat{\lambda}(A)=\inf \{h(U): U \text { is open and } A \subseteq U\}
$$


We want to show that $\hat{\lambda}$ is an outer measure. From the definition (and also item 2 in Proposition 2.3.9, it can be seen that $\hat{\lambda}$ is non-negative, monotone, and that $\hat{\lambda}(\emptyset)=0$. All that remains is to show that it is countably subadditive.

Lemma 2.3.11. The function $\hat{\lambda}$ is an outer measure.

Proof. As mentioned, we need only check that $\hat{\lambda}$ is countably subadditive. We start by showing this for open sets.

So let $U_{i}$ be a sequence of open sets, and let $U=\bigcup_{i=1}^{\infty} U_{i}$. Let $K$ be any compact subset of $U$. This means that $K \subseteq \bigcup_{i=1}^{n} U_{i}$ for some $n$. Furthermore, by Corollary 2.1.4 it follows that there are compact sets $K_{i} \subseteq U_{i}$ so that $K=\bigcup_{i=1}^{n} K_{i}$. Using item 6 from Proposition 2.3.9 and the definition of $\hat{\lambda}$ on open sets, we get that

$$
h(K) \leq \sum_{i=1}^{n} h\left(K_{i}\right) \leq \sum_{i=1}^{n} \hat{\lambda}\left(U_{i}\right) \leq \sum_{i=1}^{\infty} \hat{\lambda}\left(U_{i}\right)
$$

Since this holds for every compact $K \subseteq U$, it follows then that

$$
\hat{\lambda}(U) \leq \sum_{i=1}^{\infty} \hat{\lambda}\left(U_{i}\right)
$$

So $\hat{\lambda}$ is countably subadditive on open sets.

Now take some arbitrary sequence of sets $A_{i}$, and let $A=\bigcup_{i=1}^{\infty} A_{i}$. In the case where $\sum_{i=1}^{n} \hat{\lambda}\left(A_{i}\right)=\infty$, subadditivity follows trivially. So suppose that this sum is finite. Let $\varepsilon>0$. For each $i$, choose some $U_{i}$ so that $A_{i} \subseteq U_{i}$ and

$$
\hat{\lambda}\left(U_{i}\right) \leq \hat{\lambda}\left(A_{i}\right)+\frac{\varepsilon}{2^{n}}
$$

Let $U=\bigcup_{i=1}^{\infty} U_{i}$. It then follows that

$$
\hat{\lambda}(A) \leq \hat{\lambda}(U) \leq \sum_{i=1}^{\infty} \hat{\lambda}\left(U_{i}\right) \leq \sum_{i=1}^{\infty} \hat{\lambda}\left(A_{i}\right)+\varepsilon
$$

Since this holds for every $\varepsilon>0$, the countable subadditivity of $\hat{\lambda}$ follows. Hence $\hat{\lambda}$ is indeed an outer measure. 
Next we show that the Borel sets are $\hat{\lambda}$ measurable. So take any open subset $U$ and some arbitrary subset $A$, and suppose $\hat{\lambda}(A)<\infty$. By subadditivity we have

$$
\hat{\lambda}(A) \leq \hat{\lambda}(A \cap U)+\hat{\lambda}\left(A \cap U^{c}\right)
$$

so we only need to show the other direction. Let $\varepsilon>0$. Take some open $V \supseteq A$ such that $\hat{\lambda}(V) \leq \hat{\lambda}(A)+\varepsilon$. Let $K$ be a compact subset of $V \cap U$ so that

$$
h(K)>\hat{\lambda}(V \cap U)-\varepsilon
$$

Further, we take $L$ to be compact subset of $V \cap K^{c}$, so that

$$
h(L)>\hat{\lambda}\left(V \cap K^{c}\right)-\varepsilon \geq \hat{\lambda}\left(V \cap U^{c}\right)-\varepsilon
$$

Now since $K$ and $L$ are disjoint, it follows that $h(K)+h(L)=h(K \cup L)$. This gives us that

$$
\begin{aligned}
\hat{\lambda}(A \cap U)+\hat{\lambda}\left(A \cap U^{c}\right)-3 \varepsilon & \leq \hat{\lambda}(V \cap U)+\hat{\lambda}\left(V \cap U^{c}\right)-3 \varepsilon \\
& \leq h(K)+h(L)-\varepsilon \\
& =h(K \cup L)-\varepsilon \\
& \leq \hat{\lambda}(V)-\varepsilon \\
& \leq \hat{\lambda}(A)
\end{aligned}
$$

Since $\varepsilon$ is arbitrary, we obtain that

$$
\hat{\lambda}(A \cap U)+\hat{\lambda}\left(A \cap U^{c}\right) \leq \hat{\lambda}(A)
$$

showing that $U$ is $\hat{\lambda}$-measurable. This means if we restrict $\hat{\lambda}$ to the Borel sets (call this restriction $\lambda$ ), then we get that $\lambda$ is a measure on $G$.

The last step is to check that $\lambda$ is a Haar measure. Using Proposition 2.3.9 and Definition 2.3.10, it can be checked that $\lambda \geq 0, \lambda(G)>0$, and that $\lambda$ is invariant under (left) translations. Furthermore, Definition 2.3.10 immediately gives us outer regularity. Inner regularity is similar, but one 
must note that for any compact set $K$ and open set $U \supseteq K$, we have $\lambda(U) \geq h(K)$, so that

$$
\lambda(K)=\inf \{\lambda(U): U \text { is open and } K \subseteq U\} \geq h(K)
$$

So using Definition 2.3.10, we see that $\lambda$ is inner regular. Lastly we have that $\lambda$ is finite on compact sets, as for any compact set $K$, by Proposition 2.1.7 there is an open set $U \supseteq K$ with compact closure, giving us that

$$
\lambda(K) \leq \lambda(U) \leq h(\bar{U})
$$

So $\lambda(K)$ is finite (since $h$ is finite by definition).

We have shown the existence of a Haar measure on an arbitrary locally compact group. To finish the proof of Haar's Theorem (Theorem 2.3.5), we also need to show the (almost) uniqueness of this measure.

First however, we need the following results.

Lemma 2.3.12. Let $G$ be a locally compact group, and $\lambda$ a left Haar measure. Then for any open $U$, we have $\lambda(U)>0$.

Proof. Since $\lambda$ is regular and non-trivial, there must be some compact set $K$ so that $\lambda(K)>0$. Now, the collection of sets $(x U)_{x \in G}$ is an open cover for $K$, so there is some $x_{i} \in G$, for $i=1 \ldots n$ so that $x_{i} U$ cover $K$. It follows that $\lambda(K) \leq \sum_{i=1}^{n} \lambda\left(x_{i} U\right)=n \lambda(U)$. So $\lambda(U)>0$.

Corollary 2.3.13. Let $G$ be a locally compact group and $\lambda$ a left Haar measure. For any $f \in C_{c}(G)$ where $f$ is not identically zero and $f \geq 0$, we have that $\int_{G} f \mathrm{~d} \lambda>0$.

Proof. Take some $x \in G$ so that $f(x)>\varepsilon$ for some $\varepsilon>0$. Since $f$ is continuous, there is some neighbourhood $U$ of $x$ so that $f(y)>\varepsilon$ for all $y \in U$. Then it follows that $\int_{G} f \mathrm{~d} \lambda \geq \varepsilon \lambda(U)>0$.

Finally, we complete the proof of Haar's theorem, by showing that the Haar measure is unique. 
Theorem 2.3.14. Let $\mu$ and $\nu$ be two (left) Haar measures on a locally compact group $G$. Then $\mu=c \nu$ for some positive real number $c$.

Proof. Take any $f, g \in C_{c}(G)$, with $f, g \geq 0$ and $g$ not identically zero. We claim that the ratio

$$
\frac{\int f \mathrm{~d} \mu}{\int g \mathrm{~d} \mu}
$$

only depends on $f$ and $g$ (but not $\mu$ ). Note that Corollary 2.3.13 gives us that this ratio is defined.

Now we define $h \in C_{c}(G \times G)$ by

$$
h(x, y):=\frac{f(x) g(y x)}{\int g(t x) \nu(\mathrm{d} t)}
$$

We can then use Fubini's Theorem to observe the following identity

$$
\begin{aligned}
\iint h(x, y) \nu(\mathrm{d} y) \mu(\mathrm{d} x) & =\iint h\left(y^{-1} x, y\right) \mu(\mathrm{d} x) \nu(\mathrm{d} y) \\
& =\iint h\left(y^{-1}, x y\right) \nu(\mathrm{d} y) \mu(\mathrm{d} x)
\end{aligned}
$$

We can evaluate the left side as

$$
\begin{aligned}
\iint h(x, y) \nu(\mathrm{d} y) \mu(\mathrm{d} x) & =\iint \frac{f(x) g(y x)}{\int g(t x) \nu(\mathrm{d} t)} \nu(\mathrm{d} y) \mu(\mathrm{d} x) \\
& =\int f(x) \frac{\int g(y x) \nu(\mathrm{d} y)}{\int g(t x) \nu(\mathrm{d} t)} \mu(\mathrm{d} x) \\
& =\int f(x) \mu(\mathrm{d} x)
\end{aligned}
$$

and the right side

$$
\begin{aligned}
\iint h\left(y^{-1}, x y\right) \nu(\mathrm{d} y) \mu(\mathrm{d} x) & =\iint \frac{f\left(y^{-1}\right) g\left(x y y^{-1}\right)}{\int g\left(t y^{-1}\right) \nu(\mathrm{d} t)} \nu(\mathrm{d} y) \mu(\mathrm{d} x) \\
& =\int g(x) \mu(\mathrm{d} x) \int \frac{f\left(y^{-1}\right) \nu(\mathrm{d} y)}{\int g\left(t y^{-1}\right) \nu(\mathrm{d} t)}
\end{aligned}
$$

This means that the ratio of $\int f \mathrm{~d} \mu / \int g \mathrm{~d} \mu$ is

$$
\frac{\int f \mathrm{~d} \mu}{\int g \mathrm{~d} \mu}=\int \frac{f\left(y^{-1}\right) \nu(\mathrm{d} y)}{\int g\left(t y^{-1}\right) \nu(\mathrm{d} t)}
$$


which as we can see does not depend on the choice of $\mu$. In particular this means that for any $f \in C_{c}(G)$, we have that

$$
\int f \mathrm{~d} \mu=c \int f \mathrm{~d} \nu \quad \text { where } \quad c=\frac{\int g \mathrm{~d} \mu}{\int g \mathrm{~d} \nu}
$$

for any fixed non-zero $g \in C_{c}(G)$ with $g \geq 0$. Since this holds for arbitrary $f$, it follows that $\mu=c \nu$.

\subsection{Properties of the Haar Measure}

With existence and uniqueness now established, we denote the Haar measure of a group by $\lambda$. It is important to note that $\lambda$ may be scaled by any positive constant, so we sometimes will fix it by normalising some set of finite positive measure. We cannot always normalise the entire group $G$, as although we are guaranteed to have $\lambda(G)>0$ (as $\lambda$ is non-trivial), it may be the case that $\lambda(G)=\infty$.

When $\lambda(G)$ is finite however, we will often normalise $\lambda$ so that we have $\lambda(G)=1$. In fact, we know precisely when this is the case. As it so happens, $\lambda(G)$ is finite if and only if $G$ is compact. The proof given here was adapted from [13, Theorem 29E].

Theorem 2.4.1. Let $G$ be a locally compact group with Haar measure $\lambda$. Then $G$ is compact if and only if $\lambda(G)$ is finite.

Proof. If $G$ is compact it follows immediately that $\lambda(G)$ is finite since $\lambda$ is a Radon measure. Conversely, suppose that $\lambda(G)$ is finite. Fix some compact set $K$ with $\lambda(K)>0$. Now let $N$ be any integer so that $N \lambda(K)>\lambda(G)$. If we take any $x_{1} \ldots x_{N} \in G$, the collection of sets $x_{1} K \ldots x_{N} K$ cannot be disjoint, as otherwise the total measure of the union would be greater than $\lambda(G)$.

From this, we can find some $n \leq N$ and $x_{1} \ldots x_{n} \in G$ so that $x_{1} K \ldots x_{n} K$ are disjoint, but are no longer disjoint if we add any more translations of 
$K$. In other words, for any $x \in G$, we will have that $x K \cap\left(\bigcup_{i=1}^{n} x_{i} K\right) \neq \emptyset$, so that $x \in\left(\bigcup_{i=1}^{n} x_{i} K\right) K^{-1}$. It then follows that $\left(\bigcup_{i=1}^{n} x_{i} K\right) K^{-1}=G$, showing us that $G$ must be compact.

Before we proceed with more results, we present some examples of locally compact groups and their respective Haar measures.

Example 2.4.2. The following are examples of locally compact groups and their respective Haar measures:

- Consider the group $\mathbb{R}$ with addition as the group operation and the standard topology. The Haar measure then is simply the standard Lebesgue measure on $\mathbb{R}$. Indeed, the Lebesgue measure arises from the length function on intervals of $\mathbb{R}$, which is clearly (left-)invariant. Of course, more work is required to show this holds for arbitrary sets, and to further show that it is a Radon measure, however these are well known results.

- Let $G$ be the affine group. We can consider this group as a subgroup of $2 \times 2$ matrices of the form

$$
\left(\begin{array}{ll}
a & b \\
0 & 1
\end{array}\right)
$$

where the group operation is matrix multiplication and the topology is the subspace topology inherited from $\mathbb{R}^{4}$. One can show the Haar measure of this group can be defined by

$$
\lambda(A)=\iint_{A} \frac{1}{a^{2}} \mathrm{~d} a \mathrm{~d} b
$$

Furthermore, one can also show that

$$
\lambda^{*}(A)=\iint_{A} \frac{1}{a} \mathrm{~d} a \mathrm{~d} b
$$

defines a right Haar measure (i.e. a right-invariant Radon measure) on $G$. It is of note that the measures $\lambda$ and $\lambda^{*}$ are not scalar multiples of each other and thus define two distinct measures. 
- If $G$ is a discrete group, then the Haar measure is simply the counting measure. This is not difficult to show. Firstly we have that the compact sets of a discrete group are precisely the finite sets, and thus have finite measure. Outer regularity follows immediately as all sets are open. This is true for inner regularity on finite sets as well, whereas the case for infinite sets can be shown by constructing larger and larger finite subsets.

Lastly, we have that for any $a \in G$, the mapping $x \mapsto a x$ is a bijection, so that $|a X|=|X|$ for any $X \subseteq G$, so that $|\cdot|$ is left-invariant. We note that this argument may be used to show that the counting measure is also right-invariant.

In the last two cases we gave some examples of right Haar measures. It should be unsurprising that one may modify the proof of Haar's theorem to work for right Haar measures, by changing the covering number in Definition 2.3.6 to use right translations instead.

However, with the results we have for the left Haar measures, we can prove Haar's theorem for right Haar measures almost directly. The key idea is the following definition. If we take any Borel measure $\mu$, then we define the measure $\mu^{*}$ by

$$
\mu^{*}(A):=\mu\left(A^{-1}\right)
$$

Proposition 2.4.3. Let $G$ be a locally compact group and $\mu$ a Borel measure on $G$. We have that $\mu$ is a left/right Haar measure if and only if $\mu^{*}$ is a right/left Haar measure respectively.

Proof. We first note that $\left(\mu^{*}\right)^{*}=\mu$. Now, the mapping $x \mapsto x^{-1}$ is a homeomorphism of $G$, so that if $\mu$ is a Radon measure, we know that $\mu^{*}$ is as well (and the reverse is true as well). This means we only need to check left/right-invariance.

So suppose $\mu$ is a left Haar measure and hence left-invariant. Then

$$
\mu^{*}(A x)=\mu\left((A x)^{-1}\right)=\mu\left(x^{-1} A^{-1}\right)=\mu\left(A^{-1}\right)=\mu^{*}(A)
$$


so $\mu^{*}$ is right-invariant, and hence a right Haar measure. The same argument applies in the reverse direction, and when $\mu$ is a right Haar measure.

Corollary 2.4.4. Let $G$ be a locally compact group. Then there exists a unique (up to a constant multiple) right Haar measure $\mu$ on $G$.

As we have seen in Example 2.4.2, there are cases where the left and right Haar measures are distinct (as it is for the affine group), and there are instances where they coincide (such as any discrete group). In the latter case, such groups are called unimodular, and we shall present a way to characterise such groups.

To do this, we first need to define the following. If $G$ is a locally compact group with (left) Haar measure $\lambda$, then we let $\lambda_{x}$ be the measure defined by

$$
\lambda_{x}(A):=\lambda(A x)
$$

It is not difficult to see that $\lambda_{x}$ is a Haar measure. Indeed, we have that $\lambda_{x}(y A)=\lambda(y A x)=\lambda(A x)=\lambda_{x}(A)$, so $\lambda_{x}$ is left-invariant. Moreover, the mapping $y \mapsto y x$ is a homeomorphism of $G$, so $\lambda_{x}$ will be a Radon measure, and is therefore a Haar measure.

However, we know that the Haar measure is unique up to a constant scaling factor. In other words, there is some positive constant $c \in \mathbb{R}$ so that $\lambda_{x}=c \lambda$. We use this to define the following.

Definition 2.4.5. Let $G$ be a locally compact group with Haar measure $\lambda$. Then we define the modular function $\Delta$ as a mapping from $G$ to $\mathbb{R}^{+}$, so that for every $x \in G$, and Borel $A \subseteq G$, we have

$$
\lambda_{x}(A)=\lambda(A x)=\Delta(x) \lambda(A)
$$

The modular function is independent of our choice of Haar measure. Moreover, we can show that this function has several nice properties which will be useful to us. Perhaps the most important of these is that this function is a continuous homomorphism. The argument we present here is a modified version of the proof given by [10] 
Proposition 2.4.6. Let $G$ be a locally compact group, and $\Delta$ its modular function. Then $\Delta$ is a continuous (group) homomorphism from $G$ to $\mathbb{R}^{+}$, where $\mathbb{R}^{+}$has a group operation of multiplication, and the usual topology inherited from $\mathbb{R}$.

Proof. We first see that $\Delta$ is a group homomorphism as

$$
\Delta(x y) \lambda(A)=\lambda(A x y)=\Delta(y) \lambda(A x)=\Delta(x) \Delta(y) \lambda(A)
$$

Next we show that $\Delta$ is continuous at the identity. For this we fix some compact set $K$ with non-empty interior, and let $\varepsilon>0$. By outer regularity of $\lambda$, there is an open set $U$ so that $K \subseteq U$ and $\lambda(U)<(1+\varepsilon) \lambda(K)$. By Proposition 2.2.5, there is an open neighbourhood of the identity $V$ such that $K V \subseteq U$. Now, we have that $V \cap V^{-1}$ is also a (non-empty) open neighbourhood of the identity. So take $x \in V \cap V^{-1}$ so that

$$
\Delta(x)=\frac{\lambda(K x)}{\lambda(K)} \leq \frac{\lambda(K V)}{\lambda(K)} \leq \frac{\lambda(U)}{\lambda(K)}<1+\varepsilon
$$

We can apply the above inequality to $x^{-1}$ as well, and since $\Delta$ is a group homomorphism, we have that

$$
\Delta(x)=\frac{1}{\Delta\left(x^{-1}\right)}>\frac{1}{1+\varepsilon}
$$

Thus we obtain that $\Delta$ is continuous at the identity. We can further see that it is continuous everywhere by using the group homomorphism property.

As mentioned previously, one special class of locally compact groups are the unimodular groups. These are groups where the left and right Haar measures coincide. We can formulate this as a property of $\Delta$.

Definition 2.4.7. Let $G$ be a locally compact group. We say $G$ is unimodular if $\Delta(x)=1$ for every $x \in G$.

In particular if $\Delta(x)=1$ at every point $x \in G$, then we have that $\lambda_{x}=$ $\lambda$. This means that $\lambda$ is preserved under right translations, and thus is 
also a right Haar measure. So it is clear that left and right Haar measures coincide in unimodular groups (and the converse holds as well).

From the definition of $\Delta$, we can see that every commutative group is trivially unimodular. The same is also true for compact groups, though this requires a bit more work.

Proposition 2.4.8. Let $G$ be a locally compact group. If $G$ is compact, then $G$ is unimodular.

Proof. Since $\Delta$ is a homomorphism, it follows that $\Delta\left(x^{n}\right)=\Delta(x)^{n}$ for every $x \in G$ and $n \in \mathbb{N}$. This means that if there is some $x$ so that $\Delta(x)>1$, then $\Delta$ is unbounded. Similarly if $\Delta(x)<1$, then $\Delta\left(x^{-1}\right)=\Delta(x)^{-1}>1$, and so again $\Delta$ is unbounded.

Now, we know that $G$ is compact. Since $\Delta$ is continuous, it follows that the image of $G$ under $\Delta$ must also be compact, and so is bounded. By the above argument, this means that there is no element $x \in G$ so that either $\Delta(x)>1$ or $\Delta(x)<1$. In other words, $G$ is unimodular.

We also saw that in Example 2.4.2 that all discrete groups are also unimodular. This means that we have that all groups which are either abelian, compact, or discrete, will be unimodular. However, the converse of this statement is not true. For instance one may take $\mathbb{R} \times S_{3}$, where $S_{3}$ is the (discrete) group of permutations on three objects. As $S_{3}$ is not abelian, then neither is this product group. Furthermore, this group is not compact nor discrete as $\mathbb{R}$ is neither of these. However both groups are unimodular so it is easy to verify that their product will be as well.

\subsection{Haar Integral}

As with any measure, we can use it to construct an integral on our measurable real and even complex valued functions. This construction follows the usual method of approximation by simple functions. We shall omit this construction here, though one may look at Cohn [1, Chapter 2] for more 
details. We have in fact already used this integral in Theorem 2.3.14 to show that Haar measures are unique. For convenience, we shall denote its differential by $\mathrm{d} x$, so that $\int f \mathrm{~d} x:=\int f \mathrm{~d} \lambda$.

We previously used the modular function to evaluate the measure of a right translation of any set. We can use this to evaluate the integral of right translations of functions as well. For characteristic functions, we have that

$$
\int \chi_{A}\left(t x^{-1}\right) \mathrm{d} t=\int \chi_{A x}(t) \mathrm{d} t=\lambda(A x)=\Delta(x) \lambda(A)=\Delta(x) \int \chi_{A}(t) \mathrm{d} x
$$

We can use this to generalise this fact as for any measurable function $f$ we get

$$
\int f\left(t x^{-1}\right) \mathrm{d} t=\Delta(x) \int f(t) \mathrm{d} t
$$

The first theorem we present here gives us a nice application of the modular function which allows us to evaluate certain integrals.

Theorem 2.5.1. For any integrable function $f: G \rightarrow \mathbb{C}$, we have that

$$
\int f(x) \mathrm{d} x=\int f\left(x^{-1}\right) \Delta\left(x^{-1}\right) \mathrm{d} x
$$

Proof. We first consider the case where $f$ is the characteristic function of some Borel set $A$. With this in consideration and using eq. 2.5, we define a new measure $\mu$ by

$$
\mu(A):=\int_{A} \Delta\left(x^{-1}\right) \mathrm{d} x
$$

We claim that $\mu^{*}=\lambda$. We will show this by first showing that $\mu$ is a right Haar measure, and then using uniqueness to prove our claim

Firstly we see that $\mu$ is clearly a positive measure - this follows from the fact that $\Delta$ is positive. To show that $\mu$ is a right Haar measure, we need to prove that it is regular, finite on compact sets, and right-invariant.

Right-invariance follows from a straightforward computation. Using 
eq. 2.4), we see that for any Borel $A \subseteq G$ and $x \in G$ we have

$$
\begin{aligned}
\mu(A x) & =\int \chi_{A x}(y) \Delta\left(y^{-1}\right) \mathrm{d} y \\
& =\int \chi_{A}\left(y x^{-1}\right) \Delta\left(y^{-1}\right) \mathrm{d} y \\
& =\Delta(x) \int \chi_{A}(y) \Delta\left((y x)^{-1}\right) \mathrm{d} y \\
& =\int_{A} \Delta\left(y^{-1}\right) \mathrm{d} y=\mu(A)
\end{aligned}
$$

Next we can also see that $\mu$ is finite on compact sets. For any compact set $K$, we have that $\Delta$ is bounded on $K$, and furthermore we have $\lambda(K)$ is finite. It follows then that $\mu(K)$ is finite.

Lastly we need to show that $\mu$ is both inner and outer regular. For this we define the open set

$$
G_{n}=\left\{x \in G: \frac{1}{n}<\Delta\left(x^{-1}\right)<n\right\}
$$

Take any open set $U$. We claim that

$$
\mu\left(U \cap G_{n}\right)=\sup \left\{\mu(K): K \subseteq U \cap G_{n} \text { and } K \text { is compact }\right\}
$$

By inner regularity of $\lambda$, choose any increasing sequence of compact sets $K_{i} \subseteq U \cap G_{n}$ such that $\lim _{i \rightarrow \infty} \lambda\left(K_{i}\right)=\lambda\left(U \cap G_{n}\right)$. In the case where $\lambda\left(U \cap G_{n}\right)$ is finite, this tells us that the set $N:=\left(U \cap G_{n}\right) \backslash \bigcup K_{i}$ must be a $\lambda$ null set. Taking the limit of $\mu\left(K_{i}\right)$ and invoking the monotone convergence theorem we find

$$
\begin{aligned}
\lim _{i \rightarrow \infty} \mu\left(K_{i}\right) & =\lim _{i \rightarrow \infty} \int_{K_{i}} \Delta\left(x^{-1}\right) \mathrm{d} x \\
& =\int \lim _{i \rightarrow \infty} \chi_{K_{i}}(x) \Delta\left(x^{-1}\right) \mathrm{d} x \\
& =\int_{\left(U \cap G_{n}\right) \backslash N} \Delta\left(x^{-1}\right) \mathrm{d} x \\
& =\int_{U \cap G_{n}} \Delta\left(x^{-1}\right) \mathrm{d} x \\
& =\mu\left(U \cap G_{n}\right)
\end{aligned}
$$


Otherwise if $\lambda\left(U \cap G_{n}\right)=+\infty$, we can easily check that $\mu\left(U \cap G_{n}\right)=+\infty$. Namely, we can find an increasing sequence of compact sets $K_{i}$ so that we have $\lambda\left(K_{i}\right) \geq i$. This gives us that

$$
\mu\left(K_{i}\right)=\int_{K_{i}} \Delta\left(x^{-1}\right) \mathrm{d} x \geq i / n
$$

and so in either case we get that eq. 2.6 holds. As we let $n \rightarrow \infty$, we obtain

$$
\mu(U)=\sup \{\mu(K): K \subseteq U \text { and } K \text { is compact }\}
$$

showing us that $\mu$ is inner regular.

Now we take any arbitrary Borel set $A$. Outer regularity follows immediately if $A$ is $\mu$-infinite, so let us suppose it is $\mu$-finite. Let $\varepsilon>0$ and $n \in \mathbb{N}$. Using the outer regularity of $\lambda$, we can choose an open set $U_{n} \subseteq G_{n}$ so that $A \cap G_{n} \subseteq U_{n}$ and that $\lambda\left(U_{n}\right) \leq \lambda\left(A \cap G_{n}\right)+\varepsilon /\left(n 2^{n}\right)$. From this we obtain

$$
\begin{aligned}
\mu\left(U_{n}\right) & =\int_{U_{n}} \Delta\left(x^{-1}\right) \mathrm{d} x \\
& =\int_{A \cap G_{n}} \Delta\left(x^{-1}\right) \mathrm{d} x+\int_{U_{n} \backslash\left(A \cap G_{n}\right)} \Delta\left(x^{-1}\right) \mathrm{d} x \\
& \leq \mu\left(A \cap G_{n}\right)+\frac{\varepsilon}{2^{n}}
\end{aligned}
$$

It then follows that if we let $U=\bigcup U_{n}$, then $A \subseteq U$ with $\mu(U) \leq \mu(A)+\varepsilon$, thus showing the outer regularity of $\mu$.

With this, we have shown that $\mu$ is a right Haar measure. We know that this means that $\mu^{*}$ must therefore be a left Haar measure. By uniqueness, it follows that there is some positive constant $c$ so that $\mu^{*}(A)=c \lambda(A)$ for every Borel set $A \subseteq G$. For any $\varepsilon>0$, we can take $A$ to be a sufficiently small symmetric neighbourhood of $e$, so that $|\Delta(x)-1|<\varepsilon$ for every $x \in A$. From this we have that

$$
|c-1|=\left|\frac{\mu^{*}(A)}{\lambda(A)}-1\right|=\frac{\left|\int_{A^{-1}}\left(\Delta\left(x^{-1}\right)-1\right) \mathrm{d} x\right|}{\lambda(A)}<\frac{\lambda(A) \varepsilon}{\lambda(A)}=\varepsilon
$$

Thus it follows that $c=1$, and so $\mu^{*}=\lambda$. 
We now have that eq. 2.5 holds for all characteristic functions. However we know that integrals of arbitrary measurable functions are defined in terms of the integrals of characteristic functions. Since this equation is preserved under finite linear combinations, and also under taking limits, it follows that it must hold for any arbitrary integrable $f$.

One may consider the mapping $f \mapsto f^{*}$ where $f^{*}(x)=\overline{f\left(x^{-1}\right)} \Delta\left(x^{-1}\right)$. This mapping is an involution on $L^{1}(G)$, and the above theorem tells us that this involution is norm-preserving. Here we define $L^{1}(G)$ (and more generally $\left.L^{p}(G)\right)$ to be the usual Lebesgue space with respect to the Haar measure. Recall that for a measurable function $f: G \rightarrow \mathbb{C}$, we define the p-norm

$$
\|f\|_{p}:=\left(\int_{G}|f(x)|^{p} \mathrm{~d} x\right)^{1 / p}
$$

We then construct $L^{p}(G)$ by first constructing equivalence classes on the set of functions whose $p$-norm is finite. We say two functions $f, g$ are equivalent if $\|f-g\|_{p}=0$. We then take this set of equivalence classes, and this gives us the space $L^{p}(G)$. Some authors will explicitly differentiate between the space of functions with finite $p$-norm and $L^{p}(G)$. For the sake of simplicity and ease of presentation, we shall ignore this detail, and treat each element of $L^{p}(G)$ as a function on $G$.

We can also define $L^{\infty}(G)$, the space of essentially bounded functions. In a discrete group this is simply the space of bounded functions. Otherwise this is the space of functions which are bounded up to an exceptional locally null set.

Definition 2.5.2. We define the essential supremum of a function to be

$$
\operatorname{essup}_{x \in G} f(x):=\inf \left\{\sup _{x \in G \backslash N}|f(x)|: N \subseteq G \text { is locally null }\right\}
$$

The essential infimum is defined in a similar manner.

We then define the norm $\|f\|_{\infty}:=\operatorname{ess}_{\sup _{x \in G}}|f|$, and we can then use the same construction as above to define $L^{\infty}(G)$. 
As hinted at previously, one particularly important Lebesgue space that we shall need is $L^{1}(G)$. We can turn this space into an algebra, or more specifically a Banach $*$-algebra, by introducing a multiplication operation. This multiplication takes the form of a convolution and is defined as follows

$$
(f * g)(x):=\int f(y) g\left(y^{-1} x\right) \mathrm{d} y
$$

We can see this is indeed an element of $L^{1}(G)$ as

$$
\begin{aligned}
\|f * g\|_{1} & =\int\left|\int f(y) g\left(y^{-1} x\right) \mathrm{d} y\right| \mathrm{d} x \\
& \leq \iint\left|f(y) g\left(y^{-1} x\right)\right| \mathrm{d} y \mathrm{~d} x \\
& =\iint\left|f(y) g\left(y^{-1} x\right)\right| \mathrm{d} x \mathrm{~d} y \\
& =\iint|f(y) g(x)| \mathrm{d} x \mathrm{~d} y \\
& =\int|f(y)| \mathrm{d} y \int|g(x)| \mathrm{d} x=\|f\|_{1}\|g\|_{1}
\end{aligned}
$$

We note that we need to invoke Fubini's theorem. We need it to both swap the integrals, as well as to show that function defined by $f * g$ is indeed measurable. However, to use this theorem, we typically require measures to be $\sigma$-finite (and not all Haar measures are $\sigma$-finite). We can circumvent this issue by restricting our space to the support of $f$ and $g$ (which is necessarily $\sigma$-finite). We also note that if we add the extra condition that $f$ and $g$ are positive (in the sense that $f(x), g(x) \geq 0$ for every $x \in G$ ), then we obtain equality, so that $\|f * g\|_{1}=\|f\|_{1}\|g\|_{1}$.

We shall omit the proof that $L^{1}(G)$ with these operations is a Banach $*$ algebra, and instead refer the reader to Cohn [1, Section 9.4] for the details.

Another important space we shall need is the space of complex-valued Borel regular measures, which we denote as $M(G)$. There is a natural embedding of $L^{1}(G)$ into $M(G)$, where for any $f \in L^{1}(G)$, we define $\mu_{f} \in M(G)$ by

$$
\mu_{f}(A):=\int_{A} f(x) \mathrm{d} x
$$


With this in mind, we can extend the notions of convolution and involution to $M(G)$.

$$
\begin{aligned}
(\mu * \nu)(A) & :=(\mu \times \nu)(\{(x, y) \in G \times G: x y \in A\}) \\
& =\int \nu\left(x^{-1} A\right) \mathrm{d} \mu(x) \\
& =\int \mu\left(A y^{-1}\right) \mathrm{d} \nu(u)
\end{aligned}
$$

We can easily see that this is simply an extension of our previous definition, so that $\mu_{f} * \mu_{g}=\mu_{f * g}$. Likewise we define the involution

$$
\mu^{*}(A)=\mu\left(A^{-1}\right)
$$

We briefly mention here that $M(G)$ is isometrically linear homeomorphic to the dual of $C_{0}(G)$. This result is known as the Riesz-Markov Representation Theorem. This is a non-trivial result and we shall not prove it here. However this result will be important in the following chapters.

We can also impose a lattice structure to some elements of these spaces, via the notion of a join (least upper bound), which is denoted by $\vee$. For real-valued functions of $L^{1}(G)$, this can be simply thought of as a pointwise supremum (up to a locally null set). That is, for any $f, g \in L^{1}(G)$, we have

$$
(f \vee g)(x):=\max \{f(x), g(x)\}
$$

for all $x \in G$. It is not difficult to verify that $f \vee g \in L^{1}(G)$ where we have $\|f \vee g\| \leq\|f\|+\|g\|$

For $M(G)$ however, this definition is not as straightforward. We again only define this join for real-valued measures on this space. Since $M(G)=$ $C_{0}(G)^{*}$, we want our join to respect the structure of the predual. So for $\mu, \nu \in M(G)$, and Borel $A \subseteq G$, we define

$$
(\mu \vee \nu)(A):=\sup \{\mu(X)+\nu(A-X): X \text { is Borel and } X \subseteq A\}
$$

We again have that $\mu \vee \nu \in M(G)$. 
This additional structure also allows us to define positive and negative parts of a function:

$$
f^{+}:=f \vee 0 \quad f^{-}:=(-f) \vee 0
$$

so that we have $f=f^{+}-f^{-}$. We define this for measures in the same way. It is of note that this definition for measures coincides with the positive and negative measures obtained via the Hahn-Jordan decomposition theorem.

We can also use these parts of a function to define its absolute value. We define

$$
|f|:=f^{+}+f^{-}
$$

For functions, this corresponds to the usual definition of $|f|$, where we have $|f|(x)=|f(x)|$. However, this is not the case for measures (so in general, $|\mu|(A) \neq|\mu(A)|)$. More importantly, we can use this to define a norm, where

$$
\|\mu\|:=|\mu|(G)
$$

We note that this norm is only defined for real-valued measures. It is possible to define a norm for complex-value measures, however we shall for simplicity omit this detail.

Using this definition of norm and the involution operations previously mentioned, we can turn $M(G)$ into a Banach $*$-algebra. We again omit this proof, the details of which can also be found in Cohn [1, Section 9.4].

We can also consider taking the product of a function and a measure. For $\mu \in M(G)$ and $f \in L^{1}(G)$.

$$
(\mu * f)(x)=\int f\left(y^{-1} x\right) \mathrm{d} \mu(y)
$$

and

$$
(f * \mu)(x)=\int f\left(x y^{-1}\right) \Delta\left(y^{-1}\right) \mathrm{d} \mu(y)
$$

We shall omit the calculations here, but one may verify that these products are elements of $L^{1}(G)$. In particular we have $\|\mu * f\|,\|f * \mu\| \leq\|\mu\|\|f\|$, with equality holding when both $\mu$ and $f$ are positive. 
One particularly important measure we need here is the Dirac point measure $\delta_{x}(A)$ (where $x \in G$ ), which is 1 precisely when $x \in A$, and 0 otherwise. This means that for any $x \in G$ and $f \in L^{1}(G)$, we have $\int f \mathrm{~d} \delta_{x}=f(x)$. If we use these in our product, we get that

$$
\begin{aligned}
& (x * f)(y):=\left(\delta_{x} * f\right)(y)=\int f\left(z^{-1} y\right) \mathrm{d} \delta_{x}(z)=f\left(x^{-1} y\right) \\
& (f * x)(y):=\left(f * \delta_{x}\right)(y)=\int f\left(y z^{-1}\right) \Delta\left(z^{-1}\right) \mathrm{d} \delta_{x}(z)=f\left(y x^{-1}\right) \Delta\left(x^{-1}\right)
\end{aligned}
$$

This gives us a convenient way to define translations of functions (and measures), using convolution. We shall use the notation frequently throughout the remainder of this thesis.

One useful result that we previously alluded to is that we can embed $L^{1}(G)$ into $M(G)$. This embedding preserves all the structure properties we have discussed here. Recall that for any given $f \in L^{1}(G)$, we define $\mu_{f} \in M(G)$ by

$$
\mu_{f}(A):=\int_{A} f(x) \mathrm{d} x
$$

Indeed this definition gives rise to the following important theorem.

Theorem 2.5.3. The mapping $f \mapsto \mu_{f}$ as defined above is a linear embedding of $L^{1}(G)$ to $M(G)$. Moreover this mapping preserves the norm, joins, and translations.

Proof. We omit the details showing that $\mu_{f} \in M(G)$. However it should be clear that this indeed defines a measure, and one could verify that is a Borel regular measure. It is however clear that this mapping is linear, and so is a linear embedding.

To show it preserves the lattice structure (joins), we first take any $f \in$ $L^{1}(G)$. It is clear that $\mu_{f}=\mu_{f^{+}}-\mu_{f^{-}}$, where both $\mu_{f^{+}}$and $\mu_{f^{-}}$are positive. Now let $P=\operatorname{supp} f^{+}$, and define $N=G \backslash P$. It is not difficult then to verify that $\mu_{f^{+}}(N)=0$ and $\mu_{f^{-}}(P)=0$, thus showing that act on two different components of $G$. In particular, this gives us that $\mu_{f^{+}}$and $\mu_{f^{-}}$form a 
Hahn-Jordan decomposition of $\mu_{f}$. Since this decomposition is unique, it must follow that $\mu_{f^{+}}=\mu_{f}^{+}$and $\mu_{f^{-}}=\mu_{f}^{-}$.

We can now use this to show that our mapping preserves joins. We note that this mapping will take real-value functions to real-valued measures. If we take any real-valued $f, g \in L^{1}(G)$, then we know that

$$
\mu_{f \vee g}-\mu_{g}=\mu_{(f-g) \vee 0}=\mu_{(f-g)^{+}}=\mu_{(f-g)}^{+}=\mu_{(f-g)} \vee 0=\left(\mu_{f} \vee \mu_{g}\right)-\mu_{g}
$$

Hence $\mu_{f \vee g}=\mu_{f} \vee \mu_{g}$, thus showing that the embedding $f \mapsto \mu_{f}$ preserves joins. We can also use this to see it is norm preserving, as for every $f \in$ $L^{1}(G)$ we have

$$
\begin{aligned}
\left\|\mu_{f}\right\| & =\left|\mu_{f}\right|(G) \\
& =\mu_{f}^{+}(G)+\mu_{f}^{-}(G) \\
& =\mu_{f^{+}}(G)+\mu_{f^{-}}(G) \\
& =\int_{G}|f|(x) \mathrm{d} x=\|f\|
\end{aligned}
$$

Lastly we would like to show that this mapping preserves translations. In particular we want to show that $s * \mu_{f}=\mu_{s * f}$ for every $f \in L^{1}(G)$ and $s \in G$. So take $f \in L^{1}(G)$ and some $s \in G$. Then for any $h \in C_{0}(G)$

$$
\begin{aligned}
\left\langle s * \mu_{f}, h\right\rangle & =\int h \mathrm{~d}\left(s * \mu_{f}\right) \\
& =\iint h(x y) \mathrm{d} \delta_{s}(x) \mathrm{d} \mu_{f}(y) \\
& =\int h(s y) \mathrm{d} \mu_{f}(y) \\
& =\int h(s y) f(y) \mathrm{d} y \\
& =\int h(y) f\left(s^{-1} y\right) \mathrm{d} y \\
& =\int h(y)(s * f)(y) \mathrm{d} y \\
& =\int h \mathrm{~d} \mu_{s * f}=\left\langle\mu_{s * f}, h\right\rangle
\end{aligned}
$$

Since this holds for all $h$, it follows that $s * \mu_{f}=\mu_{s * f}$. 


\section{Chapter 3}

\section{Amenability}

\subsection{Paradoxical Decompositions}

We begin this chapter by presenting the infamous Banach-Tarski Paradox, and briefly investigating what are the necessary requirements for such a paradox to occur. The Banach-Tarski Paradox can be stated as the following theorem.

Theorem 3.1.1 (Banach-Tarski Paradox). Let $S_{2}$ be the unit sphere in three dimensions. Then there is a partition $A_{1}, A_{2}, A_{3}, A_{4}, A_{5}$ of $S_{2}$ and rigid transformations $g_{1}, g_{2}, g_{3}, g_{4}, g_{5} \in S E(3)$ such that:

- The sets $g_{1}\left(A_{1}\right), g_{2}\left(A_{2}\right), g_{3}\left(A_{3}\right)$ are disjoint.

- $g_{1}\left(A_{1}\right) \cup g_{2}\left(A_{2}\right) \cup g_{3}\left(A_{3}\right)=S_{2}$.

- The sets $g_{4}\left(A_{4}\right), g_{5}\left(A_{5}\right)$ are disjoint.

- $g_{4}\left(A_{4}\right) \cup g_{5}\left(A_{5}\right)=S_{2}$.

Here, the set of rigid transformations $S E(3)$ is the set of all rotations, translations, and combinations of both (in three dimensions).

To summarise informally, this theorem tells us that we can take the unit sphere, split it into finitely many pieces, then using only translations and rotations, reassemble these pieces into two copies of the original sphere. 
This is a surprising result which strongly contrasts our intuition about how real three dimensional objects behave.

One resolution to this paradox is that the proof of this theorem requires the axiom of choice. In particular, the sets $A_{i}$ will not be Lebesgue measurable. This means that one could not perform such a decomposition using physical spheres, which partially explains the origin of such a paradox.

However this alone is not enough. It turns out that we cannot do such a decomposition for the unit circle $\left(S_{1}\right)$, despite the fact that it also contains non-measureable sets (assuming axiom of choice). Moreover, we can even get a modified version of this statement without choice. It was shown by Dougherty and Foreman [4] that one can make a similar sort of decomposition using sets with the property of Baire, though one must take the closure. Crucially, what this means, is that this sort of decomposition is not a result of non-measureable sets, but rather something else. In fact, this is due to a certain property of the group acting on this set, in this case $S E(3)$ (the set of proper rigid transformations). We shall formalise this property into a definition.

Definition 3.1.2. Let $G$ be a group, and let $A_{1}, \ldots, A_{n}, B_{1}, \ldots, B_{m}$ be a partition of $G$. We say that this partition is a paradoxical decomposition if there exist elements $x_{1}, \ldots, x_{n}, y_{1}, \ldots, y_{m} \in G$ so that $G=\bigcup_{i=1}^{n} x_{i} A_{i}$ and $G=\bigcup_{i=1}^{m} y_{i} B_{i}$. We say that a group $G$ is paradoxical if it has a paradoxical decomposition.

Perhaps the simplest example of a paradoxical group is the free group of rank 2. This group (denoted $F_{2}$ ) is generated by two elements $a$ and $b$, so that these two elements are independent from each other. Another way to more explicitly construct this group, is to consider the set of all finite words using the symbols $a, b, a^{-1}, b^{-1}$. We say that a word is reduced if it has no pair of adjacent letters of the form $x x^{-1}$ (or $x^{-1} x$ ). Then the group $F_{2}$ is simply the set of all reduced words, including the empty word, which we write as $e$. Naturally, the group operation is concatenation, reducing the word if necessary. 


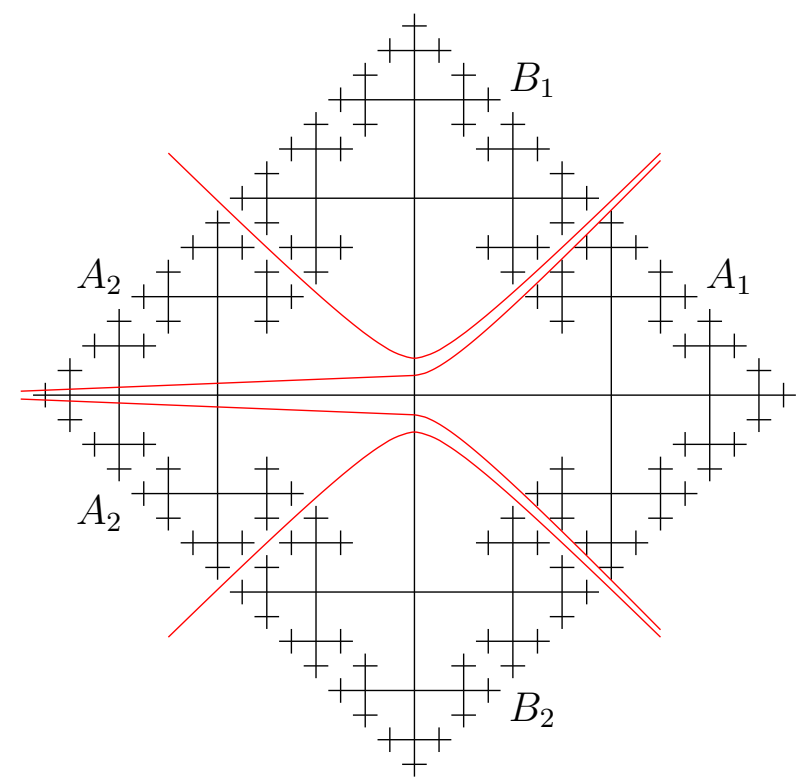

Figure 3.1: The free group $F_{2}$ with its paradoxical decomposition.

Proposition 3.1.3. The group $F_{2}$ is paradoxical.

Proof. For any reduced word $x$ in $F_{2}$, we denote $W(x)$ to be the set of all (reduced) words which begin with $x$. We shall use this to define our paradoxical decomposition as follows.

First let $A$ be the set of all words which only contain the letters $a$ and $a^{-1}$. We define our partition as follows.

$$
A_{1}=W(a) \cup A \quad A_{2}=W\left(a^{-1}\right) \backslash A \quad B_{1}=W(b) \quad B_{2}=W\left(b^{-1}\right)
$$

We can easily see these are disjoint, and it is not hard to check that they do indeed partition $F_{2}$. A visual representation of this partition is given in Figure 3.1

Next we choose $x_{1}=a^{-1}, x_{2}=e, y_{1}=b^{-1}$, and $y_{2}=e$. The crucial observation here is to see that $z^{-1} W(z)=F_{2} \backslash W\left(z^{-1}\right)$, where $z$ is any one of $a, b, a^{-1}, b^{-1}$. Furthermore, we have that $a A=a^{-1} A=A$, so it follows 
that

$$
\begin{aligned}
x_{1} A_{1} \cup x_{2} A_{2} & =\left[a^{-1} W(a) \cup a^{-1} A\right] \cup e\left[W\left(a^{-1}\right) \backslash A\right] \\
& =\left[F_{2} \backslash W\left(a^{-1}\right)\right] \cup A \cup\left[W\left(a^{-1}\right) \backslash A\right] \\
& =\left[F_{2} \backslash W\left(a^{-1}\right)\right] \cup A \cup W\left(a^{-1}\right) \\
& =F_{2} \cup A \cup W\left(a^{-1}\right) \\
& =F_{2}
\end{aligned}
$$

We also have

$$
y_{1} B_{1} \cup y_{2} B_{2}=\left[F_{2} \backslash W\left(b^{-1}\right)\right] \cup W\left(b^{-1}\right)=F_{2}
$$

and hence this partition does indeed form a paradoxical decomposition.

The existence of such a decomposition in a group is somewhat problematic when one attempts to study measures on the group. Consider a group $G$ with a paradoxical decomposition $A_{1}, \ldots, A_{n}, B_{1}, \ldots, B_{m}$. Let $\mu$ be any finitely additive, translationally invariant measure on $G$. Then we have that

$$
\mu(G)=\sum_{i=1}^{n} \mu\left(A_{i}\right)+\sum_{j=1}^{m} \mu\left(B_{j}\right)
$$

as these sets partition $G$. However, since they also form a paradoxical decomposition, then there are elements $x_{1}, \ldots, x_{n}, y_{1}, \ldots, y_{m} \in G$ so that $G=\bigcup_{i=1}^{n} x_{i} A_{i}$ and $G=\bigcup_{i=1}^{m} y_{i} B_{i}$. Therefore we have that

$$
\mu(G)=\mu\left(\bigcup_{i=1}^{n} x_{i} A_{i}\right) \leq \sum_{i=1}^{n} \mu\left(x_{i} A_{i}\right)=\sum_{i=1}^{n} \mu\left(A_{i}\right)
$$

and similarly

$$
\mu(G) \leq \sum_{i=1}^{n} \mu\left(B_{i}\right)
$$

By eq. (3.1), this means that $\mu(G) \geq 2 \mu(G)$. Then the only two possible solutions are either that $\mu(G)=0$ (and the measure is trivial), or $\mu(G)=\infty$. In particular we note that we cannot have a non-trivial finite measure on 
$G$. In fact, the converse is true as well, and this is result is known as Tarski's Theorem.

Theorem 3.1.4 (Tarski's Theorem). Let $G$ be a group. Then there exists a finitely additive, left-invariant measure $\mu$ on $G$ so that $\mu(G)=1$ if and only if $G$ is not paradoxical.

We omit the proof in the other (more difficult) direction, and refer the reader to Wagon's book [22][Corollary 9.2]. We use this theorem as motivation for the following definition.

Definition 3.1.5. Let $G$ be a group. We say that $G$ is amenable if there exists a finitely additive, left-invariant measure $\mu$ on $G$ so that $\mu(G)=1$.

This is an important definition. By using Theorem 3.1.4, we see that amenability is precisely what disallows paradoxical decompositions. One immediate example of amenable groups is all finite groups, where we may simply use the counting measure as our left-invariant measure. All abelian groups are also amenable, though showing this requires some work. On the other hand, we know any group with a paradoxical decomposition is not amenable. So by Proposition 3.1.3 the free group of rank 2 is not amenable. In fact, the Banach-Tarski Paradox tells us that the group $S E(3)$ is also paradoxical, so it too is not amenable.

The class of amenable groups also has nice closure properties. For instance, any subgroup of an amenable group is also amenable. This is easy to see for the case where the subgroup has non-zero measure (as one may simply take the restriction of the measure), but it is more difficult otherwise. We shall prove this and other similar results in the following section, where we shall generalise this definition of amenability.

\subsection{Left-Invariant Means}

A natural next step is to try and extend the definition of amenable groups to topological groups. In the case of discrete groups, where the topology 
is trivial (one could even consider such a group as not having any topological structure at all), we would like our new definition of amenability to coincide with Definition 3.1.5.

With this in mind, we first notice that the existence of a finitely additive left-invariant measure gives rise to a certain kind of integral. As our measure is only finitely additive, we do not have the access to all of the machinery of measure theory (such as the monotone convergence theorem). However, this integral does exist and is an example of a so called left-invariant mean.

Definition 3.2.1. For a locally compact group $G$, we define a left-invariant mean to be a linear functional $m$ on $L^{\infty}(G)$ so that:

- $m$ is left-invariant, that is $m(x * f)=m(f)$ for every $f \in L^{\infty}(G)$ and $x \in G$.

- For every $f \in L^{\infty}(G)$, we have

$$
\underset{x \in G}{\operatorname{essinf}} f(x) \leq m(f) \leq \underset{x \in G}{\operatorname{essip}} \sup f(x)
$$

We note that the last condition on $m$ is equivalent to condition that $m(1)=1$ and $m(f) \geq 0$ whenever $f \geq 0$. We can also use the term left-invariant mean to describe such means on spaces other than $L^{\infty}(G)$, most commonly on spaces such as the continuous bounded functions on $G$. Such means are defined almost identically, with the exception that the essential infimum (supremum) is replaced with the infimum (supremum).

Two particular spaces of functions that we shall need are $C B(G)$ and $U C B_{r}(G)$. The former is simply set of all functions $f: G \rightarrow \mathbb{C}$ that are both continuous and bounded. The latter is the set of all function which are right uniformly continuous and bounded. Here we say a function $f$ is right uniformly continuous if for any $\varepsilon>0$, there is an open neighbourhood $U$ of the identity such that $|f(x)-f(x y)|<\varepsilon$ for every $x \in G$ and $y \in U$.

As we have seen, all amenable groups (in the sense of Definition 3.1.5) will have a left-invariant mean by way of integration. We extend this definition to all locally compact groups. 
Definition 3.2.2. Let $G$ be a locally compact group. We say $G$ is amenable if it has a left-invariant mean on $L^{\infty}(G)$.

There are many examples of amenable groups, and perhaps the simplest are compact groups. We simply define our left-invariant mean to be $m(f)=\int_{G} f \mathrm{~d} \lambda$, where the Haar measure $\lambda$ is normalised so that $\lambda(G)=1$ (see Theorem 2.4.1). Another example of amenable groups is any abelian group. Showing that all abelian groups are amenable is less straightforward, though we shall later give a rough overview of the proof. One of the key steps in this will be to show that the discrete group of integers $\mathbb{Z}$ (under addition) is amenable.

The class of amenable groups has nice closure properties. As one would expect, amenability is preserved under subgroups, and also under homomorphisms. With this in mind we state the following theorems.

Theorem 3.2.3. Let $\pi$ be a surjective continuous homomorphism from $G$ to $H$, where both $G$ and $H$ are locally compact groups. If $G$ is amenable, then so is $H$.

Theorem 3.2.4. Let $G$ be amenable. If $H$ is a closed subgroup of $G$, then $H$ is amenable.

Theorem 3.2.5. Let $G$ be a locally compact group, and $H$ a closed normal subgroup. If both $H$ and $G / H$ are amenable, then so is $G$.

Theorem 3.2.6. Let $G$ be a group and let $\mathcal{H}$ be a collection of amenable subgroups of $G$ such that for any $H_{i}, H_{j} \in \mathcal{H}$, there is some $H_{k} \in \mathcal{H}$ so that $H_{i} \cup H_{j} \subseteq H_{k}$. Furthermore, if we have that the directed union of all these sets is $G$, (that is $G=\bigcup \mathcal{H})$, then it follows that $G$ is amenable.

These theorems give us nice properties about the class of amenable groups. Their proofs (as given in Greenleaf [8, Theorems 2.3.1 - 2.3.4]) often involve constructing a left-invariant mean on spaces other than $L^{\infty}(G)$. For instance the proofs often construct a left-invariant mean on $C B(G)$. It turns out that the existence of a left-invariant mean on either $C B(G)$ or 
$U C B_{r}(G)$ is equivalent to amenability (see Greenleaf [8, Theorem 2.2.1]). We shall use this to prove Theorems 3.2.3 and 3.2.5.

Proof of Theorem 3.2 .3 Let $m$ be a left-invariant mean on $C B(G)$. If $f \in$ $C B(H)$, then $f \circ \pi \in C B(G)$. Then it is not hard to verify then that the mapping $f \mapsto m(f \circ \pi)$ is a left-invariant mean on $C B(H)$.

Proof of Theorem 3.2.5 Take $f \in U C B_{r}(G)$, let $m_{1}$ be a left-invariant mean on $C B(H)$, and let $m_{2}$ be a left-invariant mean on $C B(G / H)$. Then we have that for any net $x_{\alpha} \in G$ where $x_{\alpha} \rightarrow x$, we get

$$
\left\|x_{\alpha} * f-x * f\right\| \rightarrow 0
$$

Thus it follows that $m_{1}\left(x_{\alpha} * f\right) \rightarrow m_{1}(x * f)$. In other words, the function $F$ defined by

$$
F(x)=m_{1}(x * f)
$$

is a continuous bounded function on $G$. Moreover it is constant on the cosets of $H$, so we can therefore treat $F$ as a function in $C B(G / H)$. With this, we define $\tilde{m}$ on $U C B_{r}(G)$ by $\tilde{m}(f)=m_{2}(F)$. One may easily check that this is a left-invariant mean, giving us that $G$ is amenable.

Perhaps more interestingly, is that we can formulate numerous other conditions that are equivalent to amenability, but on the surface may appear very different. In this thesis, we shall study one in particular that is known as the Følner condition.

\subsection{Følner Conditions}

The term 'Følner condition' is often used generally to describe one of many characterisations of amenability that are often of a combinatorial nature. The first and perhaps most important of these is referred to as the Følner condition, and was introduced by Følner in 1955 [7]. 
(FC) For every $\varepsilon>0$ and compact $K \subseteq G$, there is some Borel set $U$ so that $0<\lambda(U)<\infty$ and

$$
\lambda(x U \Delta U)<\varepsilon \lambda(U)
$$

for every $x \in K$.

The symbol $\Delta$ here denotes symmetric difference (so that $A \Delta B=(A \backslash B) \cup$ $(B \backslash A)$ ). Følner showed this condition to be equivalent to amenability in the discrete case. The non-discrete is case is less straightforward and requires some work, but was eventually proved by Namioka [16]. The main approach to this proof will be to find some intermediate statements which are also equivalent to amenability (and are also important in their own right). We shall roughly follow the proofs given in Greenleaf $[8]$ and Paterson [18].

We first introduce the following definition for convenience.

Definition 3.3.1. For a locally compact group $G$, we define the set $P(G)$ as the set of all positive functions in $L^{1}(G)$ with unit norm. That is

$$
P(G):=\left\{f \in L^{1}(G): f \geq 0,\|f\|_{1}=1\right\}
$$

We also introduce the notion of converging to left invariance. There are two variations of this definition, and we shall need both.

Definition 3.3.2. Let $f_{\alpha}$ be a net in $P(G)$. We say that $f_{\alpha}$ is convergent to left invariance if for every $x \in G$, we have that $\left\|x * f_{\alpha}-f_{\alpha}\right\|_{1} \rightarrow 0$ as $\alpha$ tends to infinity.

Definition 3.3.3. Let $f_{\alpha}$ be a net in $P(G)$. We say that $f_{\alpha}$ is convergent to topological left invariance iffor every $\phi \in P(G)$, we have that $\left\|\phi * f_{\alpha}-f_{\alpha}\right\|_{1} \rightarrow$ 0 as $\alpha$ tends to infinity.

These definitions naturally may also be extended to define convergence to right invariance, as well as also the notion of weak convergence 
(where one uses the weak-* topology instead of the norm). For our purposes however this definition will suffice. As it so happens, the existence of a sequence that is convergent to (topological) left invariance is equivalent to amenability. The following result, which we state without proof, was first introduced and proved by Day [3].

Theorem 3.3.4. Let $G$ be a locally compact group. Then the following are equivalent:

- $G$ is an amenable group

- There is a net in $P(G)$ that is convergent to left invariance.

- There is a net in $P(G)$ that is convergent to topological left invariance.

This is a powerful statement as it allows us to restate the amenability in terms of a net in $P(G)$. Finding such a net is often easier than constructing a left-invariant mean, as the dual of $L^{\infty}(G)$ is a very large space. The existence of such a net in $P(G)$ also allows us to formulate similar conditions that are also equivalent to amenability.

(R) For every $\varepsilon>0$ and compact set $C$, there is some $f \in P(G)$ so that

$$
\|x * f-f\|_{1}<\varepsilon
$$

for every $x \in C$.

The above condition is often referred to as Reiter's condition. This was introduced by Reiter [21, p. 405, equation (ii')] and was later shown to be equivalent to amenability by Hulanicki [11]. The proof we present here follows closely to his original proof.

Theorem 3.3.5. A locally compact group $G$ is amenable if and only if it satisfies condition (R)

Proof. First suppose $G$ satisfies (R), Let $J$ be the collection of all pairs $(\varepsilon, K)$ so that $\varepsilon>0$, and $K$ is a compact subset of $G$. We can apply a partial 
order on $J$ by saying $\left(\varepsilon_{1}, K_{1}\right) \leq\left(\varepsilon_{2}, K_{2}\right)$ if both $\varepsilon_{1} \geq \varepsilon_{2}$ and $K_{1} \subseteq K_{2}$ (so that in the limit, $\varepsilon$ gets very small and $K$ gets very large). Now, for any $j=(\varepsilon, K) \in J$, we take $\phi_{j} \in P(G)$ to satisfy condition item (R). That is

$$
\left\|x * \phi_{j}-\phi_{j}\right\|<\varepsilon
$$

for every $x \in K$. We can see that as we take a cluster point of this net of functions, we get that $\left\|x * \phi_{j}-\phi_{j}\right\| \rightarrow 0$ for every $x \in G$. Hence the net $\left\{\phi_{j}\right\}_{j \in J}$ will converge to left invariance. So $G$ is amenable.

In the other direction, we suppose $G$ is amenable, and take a net $\varphi_{\alpha}$ converging to topological left invariance. Take some $\varepsilon>0$, compact set $K \subseteq G$, and fix some element $\beta \in C_{c}(G)$, where $\beta$ is positive and has unit $L^{1}$-norm (so that $\beta \in P(G)$ ). We can find some sufficiently small compact neighbourhood $E$ of the identity so that

$$
\left\|\phi_{E} * \beta-\beta\right\|<\varepsilon \quad \text { and } \quad\|t * \beta-\beta\|<\varepsilon
$$

for all $t \in E$. Here $\phi_{E} \in P(G)$ denotes the normalised characteristic function of $E$. The existence of such a set can be seen by the (uniform) continuity of $\beta$. By compactness of $K$, we can find finitely many elements $x_{1}, \ldots, x_{n} \in G$ so that $K \subseteq \bigcup_{i=1}^{n} x_{i} E$. As $\varphi_{\alpha}$ converges to topological left invariance, we can find some $\varphi_{\alpha}$ so that

$$
\left\|\phi_{x_{i} E} * \varphi_{\alpha}-\varphi_{\alpha}\right\|<\varepsilon \quad \text { and } \quad\left\|\beta * \varphi_{\alpha}-\varphi_{\alpha}\right\|<\varepsilon
$$

We choose $\varphi=\beta * \varphi_{\alpha}$, which we will show satisfies $\|x * \varphi-\varphi\|<5 \varepsilon$ for all $x \in K$. To do so, we first see that any element $x \in K$ can be written as $x=x_{i} t$ for some $i=1, \ldots, n$ and $t \in E$. This means that we have

$$
\begin{aligned}
\left\|\phi_{E} * \varphi-t * \varphi\right\| & \leq\left\|\phi_{E} * \varphi-\varphi\right\|+\|\varphi-t * \varphi\| \\
& \leq\left\|\phi_{E} * \beta-\beta\right\|\left\|\varphi_{\alpha}\right\|+\|\beta-t * \beta\|\left\|\varphi_{\alpha}\right\| \\
& <2 \varepsilon
\end{aligned}
$$

where the last equality holds by eq. 3.3. From this we obtain

$$
\left\|\phi_{x_{i} E} * \varphi-x_{i} t * \varphi\right\|=\left\|\phi_{E} * \varphi-t * \varphi\right\|<2 \varepsilon
$$


Now, we can do the following

$$
\begin{aligned}
\|x * \varphi-\varphi\| & \leq\left\|x_{i} t * \varphi-\phi_{x_{i} E} * \varphi\right\|+\left\|\phi_{x_{i} E} * \varphi-\varphi\right\| \\
& <2 \varepsilon+\left\|\phi_{x_{i} E} * \varphi-\varphi\right\| \\
& \leq 2 \varepsilon+\left\|\phi_{x_{i} E} * \varphi-\phi_{x_{i} E} * \varphi_{\alpha}\right\|+\left\|\phi_{x_{i} E} * \varphi_{\alpha}-\varphi_{\alpha}\right\|+\left\|\varphi_{\alpha}-\varphi\right\| \\
& \leq 4 \varepsilon+\left\|\phi_{x_{i} E}\right\|\left\|\varphi-\varphi_{\alpha}\right\| \\
& <5 \varepsilon
\end{aligned}
$$

The final inequality is obtained by applying eq. (3.4) to each of the three normed terms. This gives us our desired inequality, and completes the proof.

This theorem is a major stepping stone in showing that condition (FC) is equivalent to amenability. To prove this we will introduce a weaker variation of the Følner condition, and simultaneously show both are equivalent to amenability. The condition is as follows:

(WFC) For any $\delta, \varepsilon>0$, and any compact set $C \subseteq G$, there are Borel sets $U, N$ so that $0<\lambda(U)<\infty, \lambda(N)<\delta$, and

$$
\lambda(x U \Delta U)<\varepsilon \lambda(U)
$$

for all $x \in C \backslash N$.

We can now prove this result.

Theorem 3.3.6. Let $G$ be a locally compact group. The following are equivalent.

- $G$ is amenable.

- $G$ satisfies (WFC).

- $G$ satisfies $(F C)$

Proof. We first show that amenability implies (WFC). Let $\varepsilon, \delta>0$, and take any compact set $C$. Since $G$ is amenable, then we have that (R) holds, so take some $f \in P(G)$, so that $\|x * f-f\|<\varepsilon \delta / \lambda(C)$ for all $x \in C$. Without 
loss of generality, we may assume that $f$ is a positive simple function, and write

$$
f=\sum_{i=1}^{n} a_{i} \phi_{A_{i}}
$$

where $A_{1} \supseteq \cdots \supseteq A_{n}$ are $\lambda$-finite, non-null Borel sets, and $a_{i}>0$. Now, we have that

$$
\sum_{i=1}^{n} a_{i} \frac{\lambda\left(x A_{i} \Delta A_{i}\right)}{\lambda\left(A_{i}\right)}=\sum_{i=1}^{n} a_{i}\left\|\phi_{x A_{i}}-\phi_{A_{i}}\right\|=\|x * f-f\|_{1}<\frac{\varepsilon \delta}{\lambda(C)}
$$

Integrating $x$ over $C$ we get

$$
\sum_{i=1}^{n} a_{i} \int_{C} \frac{\lambda\left(x A_{i} \Delta A_{i}\right)}{\lambda\left(A_{i}\right)} \mathrm{d} x<\varepsilon \delta
$$

and since this is a convex sum, there must be some $A=A_{i}$ so that

$$
\int_{C} \frac{\lambda(x A \Delta A)}{\lambda(A)} \mathrm{d} x<\varepsilon \delta
$$

Now, let $N \subseteq C$ be the set of all points $x$ where $\lambda(x A \Delta A) / \lambda(A) \geq \varepsilon$. Since the value of the integral is strictly less than $\varepsilon \delta$, it then follows that size of $N$ must be $\lambda(N)<\delta$. Hence we have that

$$
\frac{\lambda(x A \Delta A)}{\lambda(A)}<\varepsilon
$$

for every $x \in C \backslash N$. So $G$ satisfies (WFC)

Now suppose $G$ satisfies (WFC), and we will aim to show that it satisfies (FC) So let $\varepsilon>0$ and let $K$ be a non-null compact set. Take $A=$ $K \cup K K$, which is compact. We can then apply (WFC) to $\varepsilon / 2, \delta=\frac{1}{2} \lambda(K)$ and the compact set $A$. So there are Borel sets $U$ and $N \subseteq A$ so that $U$ is $\lambda$-finite and non-null, $\lambda(N)<\delta$, and

$$
\lambda(x U \Delta U)<\frac{\varepsilon}{2} \lambda(U)
$$


for every $x \in A \backslash N$. For convenience we define $M=A \backslash N$. Now, for every $x \in K$, we have

$\lambda(x A \cap A)=\lambda((x K \cup x K K) \cap(K \cup K K)) \geq \lambda(x K \cap K K)=\lambda(x K)=\lambda(K)$

so it follows that

$2 \delta \leq \lambda(K) \leq \lambda(x A \cap A) \leq \lambda(x M \cap M)+\lambda(N)+\lambda(x N)<\lambda(x M \cap M)+2 \delta$

This gives us that $x M \cap M$ must be non-empty (as it is not null), and so $x \in M M^{-1}$. In other words we get that $K \subseteq M M^{-1}$.

Following on from this, if we take any $n, m \in M$, from the condition given by (WFC) we get that

$$
\begin{aligned}
\lambda\left(n m^{-1} U \Delta U\right) & =\lambda\left(m^{-1} U \Delta n^{-1} U\right) \\
& \leq \lambda\left(m^{-1} U \Delta U\right)+\lambda\left(U \Delta n^{-1} U\right) \\
& =\lambda(m U \Delta U)+\lambda(n U \Delta U) \\
& <\varepsilon \lambda(U)
\end{aligned}
$$

If we let $x=n m^{-1}$, we see that $\lambda(x U \Delta U)<\varepsilon \lambda(U)$ for all $x \in K \subseteq M M^{-1}$. Hence $G$ satisfies (FC).

Lastly, we show that (FC) implies amenability. For any $\varepsilon>0$, and compact set $K$, we know by (FC) that there is some Borel set $U$ so that for every $x \in K$, we have

$$
\lambda(x U \Delta U)<\varepsilon \lambda(U)
$$

If we let $\phi=\phi_{U}$, we see that

$$
\|x * \phi-\phi\|=\frac{1}{\lambda(U)} \lambda(x U \Delta U)<\varepsilon
$$

for all $x \in K$. Thus $G$ satisfies $(\mathrm{R})$, and is therefore amenable.

We have already seen that all compact groups are amenable. A classic example of a non-compact amenable groups is the (discrete) group of integers $\mathbb{Z}$. We can show this is amenable using Følner's condition. 
Example 3.3.7. The discrete group of integers $\mathbb{Z}$ is amenable. Take any $\varepsilon>0$ and any finite (compact) subset $C$. Let $m=\max \{|c|: c \in C\}$. Further, let $U$ be the set of all integers between $-n$ and $n$ (inclusive), where $2 n+1>2 m / \varepsilon$. It follows that for any $x \in C$ (so $|x| \leq m)$, we have

$$
\frac{|(x+U) \Delta U|}{|U|}=\frac{2|x|}{2 n+1}<2|x| \frac{\varepsilon}{2 m} \leq \varepsilon
$$

Hence $U$ satisfies Følner's condition, and so $\mathbb{Z}$ is amenable.

In fact, the very same argument can be used to show that $\mathbb{R}$ under addition is amenable as well. Furthermore, this argument can be used to show that every abelian group is amenable. We shall present a sketch proof of this theorem, as we omit a few minor details.

Theorem 3.3.8. Every abelian group is amenable.

Proof sketch. Firstly, since the direct product of two groups is amenable (Theorem 3.2.5), we see that for any integer $n$, the group $\mathbb{Z}^{n}$ is amenable. We can then use a combination of this fact and that amenability is closed under direct unions (Theorem 3.2.6 to show that any free abelian group is amenable. Here a free abelian group is defined in a similar way as to the free group - we have a basis set $X$ (which may be infinite) which is used to generate the elements of our group. Naturally, the elements of this basis are defined so that they commute with each other.

Now we take any abelian group $G$. It is not difficult to see that we can find a free abelian group $F$ so that there exists a surjective homomorphism from $F$ to $G$. We can then use Theorem 3.2 .3 to see that $G$ must be amenable. 


\section{Chapter 4}

\section{Pseudo-Amenability}

\subsection{Definition}

We introduced the Følner condition (FC) in the previous chapter, and we showed it was a characterisation of amenability. One may strengthen this condition as follows.

(SF) For every $\varepsilon>0$, and every compact $K$, there is a compact $C$ such that

$$
\lambda(K C \backslash C)<\varepsilon \lambda(C)
$$

This condition was shown to be equivalent to Følner's condition (and hence also to amenability) by Emerson and Greenleaf [5].

It is also possible to weaken the Følner condition as follows.

(WF) For every $\varepsilon>0$, there is an $n \in \mathbb{N}$ such that for every finite $F \subseteq G$ with $|F| \geq n$, there is a compact $C$ so that

$$
\lambda(E C)<\varepsilon|E| \lambda(C)
$$

for every $E \subseteq F$ with $|E| \geq n$.

This condition was introduced by Pham and was proven to be equivalent to amenability [19, Theorem 5.1]. 
One may ask if quantifying over large enough subsets of $F$ in (WF) is necessary. Removing this constraint, we obtain a condition is known as pseudo-amenability, which was introduced for discrete groups by Dales and Polyakov [2, Definition 5.5]. We restate this definition for general (nondiscrete) locally compact groups.

Definition 4.1.1. Let $G$ be a locally compact group with Haar measure $\lambda$. We say $G$ is pseudo-amenable if it satisfies the following condition.

(PA) For every $\varepsilon>0$ there is some $n \in \mathbb{N}$ such that, for every finite $F \subseteq G$ with $|F| \geq n$ there is some compact $C \subseteq G$ so that

$$
\lambda(F C)<\varepsilon|F| \lambda(C)
$$

From condition (WF), it is immediate that amenability implies pseudoamenability, however the converse still remains an open problem. One may alter condition (WF) slightly, so that instead of requiring it to hold for every $\varepsilon \in(0,1)$, we instead modify the quantifier so that it need only be true for one $\varepsilon \in(0,1)$. As it turns out, this weaker condition is still equivalent to amenability [19, Theorem 5.1]. One may weaken the condition for pseudo-amenability in a similar way.

Definition 4.1.2. Let $G$ be a locally compact group with Haar measure $\lambda$. We say $G$ is weakly pseudo-amenable if it satisfies the following condition.

(WPA) There exists some $\varepsilon \in(0,1)$ and $n \in \mathbb{N}$, so that for every finite $F \subseteq G$ with $|F| \geq n$ there is some compact $C \subseteq G$ so that

$$
\lambda(F C)<\varepsilon|F| \lambda(C)
$$

Naturally, this condition is weaker than (PA) It is an open problem as to whether this condition is equivalent to pseudo-amenability. Throughout the rest of this chapter, we focus on pseudo-amenability. However, the majority of proofs we use do not manipulate $\varepsilon$ in any way. This means 
that many of the theorems we present can be stated for weakly pseudoamenable groups as well. We shall omit the statements of these theorems and their proofs for the sake of brevity.

We briefly note here that in eqs. (4.3) and (4.4), we have that our finite set $F$ is on the left side of the product $F C$. We can then interpret these equations as saying that the size of multiple left translates of a compact set $C$ joined together is a factor of $\varepsilon$ smaller than the size of the sets individually.

In the case where our group $G$ is a discrete group, the definition of pseudo-amenability may be simplified somewhat. Namely we get that $C$ must be finite and our equation can be written as

$$
|F C|<\varepsilon|F||C|
$$

Using this formulation, we can in fact directly show that any subgroup of a discrete pseudo-amenable group is itself pseudo-amenable. The nondiscrete case will require some more work, and we shall prove it later in the chapter (see Theorem 4.3.2).

Theorem 4.1.3. Let $G$ be a discrete pseudo-amenable group. If $H$ is a subgroup of $G$, then $H$ is pseudo-amenable.

Proof. First, let $\varepsilon>0$, and take $n \in \mathbb{N}$ large enough, so that for every finite $F \subseteq G$ with $|F| \geq n$, there is a finite set $C \subseteq G$ such that

$$
|F C|<\varepsilon|F||C|
$$

Fix some $F \subseteq H$ with $|F| \geq n$.

Define $\mathcal{C}$ to be the collection of all non-empty finite sets $C$ with positive measure which satisfy eq. 4.6 above. We note that as $G$ is pseudoamenable, then $\mathcal{C}$ must be non-empty. We also note that since every set in $\mathcal{C}$ is finite, $\mathcal{C}$ must have some minimal element (though it may not be unique). Here we define minimality in terms of cardinality, so that for $A, B \in \mathcal{C}$, we have $A \leq B$ if $|A| \leq|B|$. 
Further we claim that $\mathcal{C}$ is invariant under right translations. To see this, take any $C \in \mathcal{C}$ and $x \in G$. We want to show that $C x \in \mathcal{C}$. First we note that clearly $C x$ is also compact (finite). Moreover, we will have that $|C x|=|C|$, so that

$$
|F C|<\varepsilon|F||C|
$$

which implies that

$$
|F C x|<\varepsilon|F||C x|
$$

Hence $C x \in \mathcal{C}$, and so $\mathcal{C}$ is invariant under right translation.

Now, let $C^{\prime}$ be a minimal element of $\mathcal{C}$. By eq. 4.6 we see that $C^{\prime}$ cannot be empty. So take any $c \in C^{\prime}$, and define $C=C^{\prime} c^{-1}$. Since $\mathcal{C}$ is translation invariant, we see that $C \in \mathcal{C}$. Furthermore, from this definition we see that $e_{G} \in C$, so $C \cap H$ is non-empty. We claim that, in fact, $C \subseteq H$.

We suppose toward contradiction that this is not the case (so $C \nsubseteq H$ ). First define $D=C \cap H$ and $E=C \backslash H$. By assumption, $E$ is non-empty, and we also have that $D$ is non-empty as well. Since this is the case, and $D \cup E=C$, then it follows that both are proper subsets of $C$. However since $C$ is the minimal element in $\mathcal{C}$, it follows that neither $D$ nor $E$ are in $\mathcal{C}$. In other words, we must have that

$$
\begin{aligned}
& |F D| \geq \varepsilon|F||D| \\
& |F E| \geq \varepsilon|F||E|
\end{aligned}
$$

and so

$$
|F D|+|F E| \geq \varepsilon|F|(|D|+|E|)
$$

Now, since $D$ and $E$ are disjoint, and that $D \cup E=C$, we must have $|D|+|E|=|C|$.

Further, we see that since both $F$ and $D$ are subsets of $H$, then we have $F D \subseteq H$. However, $E$ is disjoint from $H$ which gives us that $F E$ must also be disjoint from $H$. This gives us then that $F D$ and $F E$ are disjoint, and hence

$$
|F D|+|F E|=|F D \cup F E|=|F(D \cup E)|=|F C|
$$


Thus we obtain

$$
|F C| \geq \varepsilon|F||C|
$$

and we therefore have a contradiction. Hence $C \subseteq H$.

This gives us that $C$ is a compact subset of $H$, and thus 'witnesses' the pseudo-amenability of $H$ for the given $F$.

Unfortunately, this proof does not generalise to non discrete pseudoamenable groups, even if one puts the additional restriction that $H$ must be closed. For now however, we turn our attention to other similar properties of pseudo-amenable groups.

We know that amenable groups are closed under directed union. We can equivalently state this by saying that if the closure of any finitely generated subgroup is amenable, then the group itself is amenable. We have a similar result for pseudo-amenable groups, though we require it for all countably generated subgroups. Fortunately however, this proof works for a general locally compact group (not just discrete groups).

Proposition 4.1.4. Let $G$ be a locally compact group. Suppose that every densely countably generated subgroup of $G$ is pseudo-amenable. Then it follows that $G$ is pseudo-amenable.

Proof. Suppose that $G$ is not pseudo-amenable. Then by the definition, there is some $\varepsilon>0$ such that for every $n \in \mathbb{N}$ there is some finite $F_{n} \subseteq G$ with $\left|F_{n}\right| \geq n$ so that for every compact set $C$, we have

$$
\lambda\left(F_{n} C\right) \geq \varepsilon\left|F_{n}\right| \lambda(C)
$$

Let $F=\bigcup_{n \in \mathbb{N}} F_{n}$, and let $H$ be the closed subgroup generated by $F$. Note that $F$ is countable and so $H$ is densely countably generated. Now we note that $F_{n} \subseteq H$ for every $n \in \mathbb{N}$. Furthermore, for any $C \subseteq H, C$ is compact in $G$ if and only if $C$ is compact in $H$. It then immediately follows that $H$ is not pseudo-amenable. 
We can also show that amenability is preserved in opposite groups. Recall that we define $\lambda^{*}(A)=\lambda\left(A^{-1}\right)$, and that this defines a right Haar measure on $G$. Alternatively we may view this as a left Haar measure on $G^{\circ}$, the opposite group of $G$.

Definition 4.1.5. Let $G=(G, \cdot)$ be a group. We say the opposite group of $G$ is the group $G^{\circ}=(G, \circ)$ such that $x \circ y=y \cdot x$.

Using this definition, it is easy to show that $G^{\circ}$ is pseudo-amenable precisely whenever $G$ is.

Proposition 4.1.6. Let $G$ be a locally compact group. Then $G$ is pseudo-amenable if and only if $G^{\circ}$ is.

Proof. First we take any $\varepsilon$ and choose $N$ satisfying (PA). Now take any finite $F \subseteq G^{\circ}$. Choose a compact set $C$ so that

$$
\lambda\left(F^{-1} C\right)<\varepsilon\left|F^{-1}\right| \lambda(C)
$$

Now we know that $C^{-1}$ is also compact so that

$$
\lambda^{*}\left(F \circ C^{-1}\right)=\lambda^{*}\left(C^{-1} F\right)=\lambda\left(F^{-1} C\right)<\varepsilon\left|F^{-1}\right| \lambda(C)=\varepsilon|F| \lambda^{*}\left(C^{-1}\right)
$$

Hence $G^{\circ}$ satisfies (PA) The converse follows as $\left(G^{\circ}\right)^{\circ}=G$.

\subsection{Characterisations}

We now aim to show two new characterisations of pseudo-amenability. These will aid us in showing some more closure properties of the class of pseudo-amenable groups. These two characterisations are conditions (L) and (M) as given below.

(L) For every $\varepsilon \in(0,1)$, there is some $N \in \mathbb{N}$ such that for all $n \geq N$, and all $s_{1}, \ldots, s_{n} \in G$ (not necessarily distinct), there is some $f \in L^{1}(G)^{+}$ so that

$$
\left\|s_{1} * f \vee \ldots \vee s_{n} * f\right\|<\varepsilon n\|f\|
$$


(M) For every $\varepsilon \in(0,1)$, there is some $N \in \mathbb{N}$ such that for all $n \geq N$, and all $s_{1}, \ldots, s_{n} \in G$ (not necessarily distinct), there is some $\mu \in M(G)^{+}$ so that

$$
\left\|s_{1} * \mu \vee \ldots \vee s_{n} * \mu\right\|<\varepsilon n\|\mu\|
$$

The two spaces denoted in these conditions are the positive real-valued functions of $L^{1}(G)$ and the positive real-valued measures of $M(G)$ respectively. We note that apart from the difference in this space, these conditions are identical. We also note that the representatives $f$ and $\mu$ in eqs. 4.7) and 4.8 ) can be chosen so they have unit norm. This can be done by taking the function $f /\|f\|$, and showing that it preserves eq. 4.7) (and similarly for measures).

We will first prove $(\mathrm{L})$, and then use it to prove $(\mathrm{M})$. Before we can attempt this we need a few intermediate results. Firstly, due to the regularity of our Haar measure $\lambda$, it is not necessary for the set $C$ we choose in condition (PA) to be compact. The main condition on $C$ is that it must be $\lambda$-finite. Indeed, this condition is satisfied if the set we choose is an open set:

(O) For every $\varepsilon \in(0,1)$, there is some $n \in \mathbb{N}$ such that for all finite $F$ with $|F| \geq n$, there is some $\lambda$-finite open set $U$ such that

$$
\lambda(F U)<\varepsilon|F| \lambda(U)
$$

or even any Borel set will suffice:

(B) For every $\varepsilon \in(0,1)$, there is some $n \in \mathbb{N}$ such that for all finite $F$ with $|F| \geq n$, there is some $\lambda$-finite Borel set $B$ such that

$$
\lambda(F B)<\varepsilon|F| \lambda(B)
$$

We prove the equivalence of these as follows. 
Proposition 4.2.1. Let $G$ be a locally compact group. Then the following are equivalent:

- $G$ is pseudo-amenable.

- $G$ satisfies $(O)$

- $G$ satisfies (B)

Proof. To show that $\rightarrow(\mathrm{B}) \rightarrow(\mathrm{O})$, take some $\varepsilon \in(0,1)$, choose $n \in \mathbb{N}$ so that for every finite set $F$ with $|F| \geq n$, we have

$$
\lambda(F B)<\varepsilon|F| \lambda(B)
$$

for some $\lambda$-finite Borel set $B$. As the inequality is strict, we can find a $\delta>0$ so that

$$
\lambda(F B)+\delta|F|<\varepsilon|F| \lambda(B)
$$

Now, by outer regularity of the Haar measure, there is some open set $U$ so that $B \subseteq U$ and $\lambda(U)<\lambda(B)+\delta$, which we can rewrite as $\lambda(U-B)<\delta$. Since $F U-F B \subseteq F(U-B)$, it follows that

$$
\lambda(F U-F B) \leq \lambda(F(U-B)) \leq \sum_{s \in F} \lambda(s(U-B))=|F| \lambda(U-B)<|F| \delta
$$

This means that $\lambda(F U) \leq \lambda(F B)+|F| \delta$, giving us that

$$
\lambda(F U) \leq \lambda(F B)+\delta|F|<\varepsilon|F| \lambda(B) \leq \varepsilon|F| \lambda(U)
$$

which gives us condition $(\mathrm{O})$.

Next we show that $(\mathrm{O}) \rightarrow(\mathrm{PA})$ So again, let $\varepsilon \in(0,1)$ and choose $n \in \mathbb{N}$, so that for every finite set $F$ with $|F| \geq n$, we have

$$
\lambda(F U)<\varepsilon|F| \lambda(U)
$$

for some $\lambda$-finite open set $U$. As we have strict inequality, then there is some $\delta>1$ so that

$$
\lambda(F U)<\frac{\varepsilon}{\delta}|F| \lambda(U)
$$


Now, by inner regularity of $\lambda$, there is some compact set $C$ such that $C \subseteq U$ and $\lambda(U) \leq \lambda(C) \delta$. Since $C \subseteq U$, it follows that $F C \subseteq F U$. This means that

$$
\lambda(F C) \leq \lambda(F U)<\frac{\varepsilon}{\delta}|F| \lambda(U) \leq \varepsilon|F| \lambda(C)
$$

So it follows that $G$ is pseudo-amenable, thus proving our claim.

Lastly it is trivial that $(\mathrm{PA}) \rightarrow(\mathrm{B})$, as all compact sets are Borel and $\lambda$-finite.

For our next result, recall that when we define integration over a measure, this is defined in terms of simple functions, which can be written as finite sums over characteristic functions. For a given simple function $f$ we often write

$$
f=\sum_{i=1}^{n} \alpha_{i} \chi_{A_{i}}
$$

where $\alpha_{i} \in \mathbb{C}$ and $A_{i}$ are disjoint Borel sets. However we can change this slightly and write $f$ as

$$
f=\sum_{i=1}^{m} \beta_{i} \chi_{B_{i}}
$$

where here we have $\beta_{i} \in \mathbb{C}$ and $B_{i}$ are Borel sets so that $B_{1} \supseteq B_{2} \supseteq \ldots \supseteq$ $B_{m}$. We shall use this form since, as we shall see, it provides nice results when one applies translations and joins to this function. In particular, in the case where we have a positive simple function, applying these translations and joins as in condition (L) gives us another simple function that can also be easily written in this form.

Lemma 4.2.2. Let $f$ be a simple function so that $f=\sum_{i=1}^{m} \beta_{i} \chi_{B_{i}}$, where $\beta_{i}>0$ and $B_{1} \supseteq B_{2} \supseteq \ldots \supseteq B_{m}$. Let $F=\left\{s_{1}, \ldots, s_{n}\right\}$. Then we have that

$$
s_{1} * f \vee \ldots \vee s_{n} * f=\sum_{i=1}^{m} \beta_{i} \chi_{F B_{i}}
$$

Proof. Firstly for convenience, define $g=s_{1} * f \vee \ldots \vee s_{n} * f$. Fix some $x \in G$. We define $j_{x}$ to be the largest integer so that $x \in s_{k} B_{j_{x}}$ for some 
$k \in\{1, \ldots, n\}$. This means that $\left(s_{l} * f\right)(x)$ is maximal when $l=k$, and so

$$
g(x)=\left(s_{k} * f\right)(x)=\sum_{i=1}^{m} \beta_{i} \chi_{s_{k} B_{i}}(x)=\sum_{i=1}^{j_{x}} \beta_{i}
$$

Now, $j_{x}$ can also be seen to be the largest integer so that $x \in F B_{j_{x}}$. Furthermore, it is easy to check that $F B_{1} \supseteq \ldots \supseteq F B_{m}$. This means that $x \in F B_{i}$ for $i \leq j_{x}$, and $x \notin F B_{i}$ for $i>j_{x}$. So it must follow that

$$
\sum_{i=1}^{m} \beta_{i} \chi_{F B_{i}}(x)=\sum_{i=1}^{j_{x}} \beta_{i}=g(x)
$$

thus completing the proof.

In order to use the above result in a meaningful way, we shall first need to obtain a simple function which has a sort of 'pseudo-amenable property'. Fortunately, this is possible as the simple functions are dense in $L^{1}(G)$.

Lemma 4.2.3. Let $G$ be a locally compact group. Further, let $X$ be a dense subset of $L^{1}(G)^{+}$. Now fix some $s_{1}, \ldots, s_{n} \in G$, and take any $\varepsilon \in(0,1)$. If there is some $f \in L^{1}(G)^{+}$so that

$$
\left\|s_{1} * f \vee \ldots \vee s_{n} * f\right\|_{1}<\varepsilon n\|f\|_{1}
$$

then there is a $g \in X$ which also satisfies the above equation.

Proof. Firstly, we may scale $f$ so that $\|f\|_{1}=1$. Since the inequality in eq. 4.11) is strict, we can find a $\delta>0$ so that

$$
\left\|s_{1} * f \vee \ldots \vee s_{n} * f\right\|_{1}<(\varepsilon-\delta) n
$$

Now, take any $g \in X$ so that $\|f-g\|_{1}<\delta /(1+\varepsilon)$. This gives us that

$$
\|g\|_{1}>\|f\|_{1}-\|f-g\|_{1}>1-\frac{\delta}{1+\varepsilon}
$$

Furthermore, we can bound the size $s_{1} * g \vee \ldots \vee s_{n} * g$ as

$$
\left\|s_{1} * g \vee \ldots \vee s_{n} * g\right\|_{1}<n\|f-g\|_{1}+\left\|s_{1} * f \vee \ldots \vee s_{n} * f\right\|_{1}
$$


which gives us that

$$
\begin{aligned}
\left\|s_{1} * g \vee \ldots \vee s_{n} * g\right\|_{1} & <\frac{\delta n}{1+\varepsilon}+(\varepsilon-\delta) n \\
& =\varepsilon n\left(1-\frac{\delta}{1+\varepsilon}\right) \\
& <\varepsilon n\|g\|_{1}
\end{aligned}
$$

Hence $g$ satisfies eq. 4.11.

We now have the tools to prove the characterisation (L), One may notice that this condition is similar to Reiters condition (R). Indeed, we shall use an argument that is somewhat similar to the proof of Følner's condition from Reiters condition (see Theorem 3.3.6 and Greenleaf [8, p. 66]).

Theorem 4.2.4. Let $G$ be a locally compact group. Then $G$ is pseudo-amenable if and only if $G$ satisfies condition $(L)$.

(L) For every $\varepsilon \in(0,1)$, there is some $N \in \mathbb{N}$ such that for all $n \geq N$, and all $s_{1}, \ldots, s_{n} \in G$ (not necessarily distinct), there is some $f \in L^{1}(G)^{+}$so that

$$
\left\|s_{1} * f \vee \ldots \vee s_{n} * f\right\|_{1}<\varepsilon n\|f\|_{1}
$$

Proof. We start by showing that (PA) implies (L). We take $\varepsilon \in(0,1)$, and fix $N \in \mathbb{N}$ to satisfy (PA), Take any $s_{1} \ldots s_{n} \in G$ where $n \geq N$, and let $F=\left\{s_{1}, \ldots, s_{n}\right\}$. We note that if our elements $s_{1}, \ldots, s_{n}$ are not distinct, we may without loss of generality extend $F$ to a larger finite set so that $|F|=n$.

By (PA), we obtain that there is a compact set $C$ so that eq. 4.3 holds. Now, we define $f=\chi_{C}$, which gives us that $\|f\|_{1}=\lambda(C)$ and

$$
\left\|s_{1} * f \vee \ldots \vee s_{n} * f\right\|_{1}=\left\|\chi_{F C}\right\|_{1}=\lambda(F C)
$$

Thus we obtain that

$$
\left\|s_{1} * f \vee \ldots \vee s_{n} * f\right\|_{1}=\lambda(F C)<\varepsilon|F| \lambda(C)=\varepsilon n\|f\|_{1}
$$


so that $G$ has property (L)

For the other direction, we again take $\varepsilon \in(0,1)$, and choose $N \in \mathbb{N}$ so that it satisfies (L). We let $F$ be a finite subset whose elements are $s_{1}, \ldots, s_{n}$ where $n \geq N$.

Now by (L), we know that there is some $f \in L^{1}(G)^{+}$so that eq. (4.7) holds. By Lemma 4.2.3, we can without loss of generality take $f$ to be a positive simple function (as simple functions are dense in $L^{1}(G)^{+}$). Moreover we can scale $f$ so that $\|f\|_{1}=1$. As $f$ is simple we can write

$$
f=\sum_{i=1}^{m} \beta_{i} \chi_{B_{i}}
$$

where $\beta_{i}>0$ and $B_{1} \supseteq B_{2} \supseteq \ldots \supseteq B_{m}$ are Borel. We also have that $0<\lambda\left(B_{i}\right)<\infty$ for each $B_{i}$, as $\|f\|_{1}=1$. Now, by Lemma 4.2.2, we can see that

$$
\begin{aligned}
\left\|s_{1} * f \vee \ldots \vee s_{n} * f\right\|_{1} & =\left\|\sum_{i=1}^{m} \beta_{i} \chi_{F B_{i}}\right\|_{1} \\
& =\sum_{i=1}^{m} \beta_{i} \lambda\left(F B_{i}\right) \\
& =\sum_{i=1}^{m}\left(\beta_{i} \lambda\left(B_{i}\right)\right)\left(\frac{\lambda\left(F B_{i}\right)}{\lambda\left(B_{i}\right)}\right)
\end{aligned}
$$

The right hand side of this equation is a convex sum, as $\sum_{i=1}^{m} \beta_{i} \lambda\left(B_{i}\right)=$ $\|f\|_{1}=1$. This means that there must be some $B=B_{i}$ so that

$$
\left\|s_{1} * f \vee \ldots \vee s_{n} * f\right\|_{1} \geq \frac{\lambda(F B)}{\lambda(B)}
$$

It then follows that

$$
\lambda(F B) \leq\left\|s_{1} * f \vee \ldots \vee s_{n} * f\right\|_{1} \lambda(B)<\varepsilon n \lambda(B)
$$

This gives us condition (B) for $G$, and by Proposition 4.2.1 this means that $G$ is pseudo-amenable. 
From this we can directly prove the second condition (M)

Theorem 4.2.5. A group $G$ is pseudo-amenable if and only if condition (M) below is satisfied.

(M) For every $\varepsilon \in(0,1)$, there is some $N \in \mathbb{N}$ such that for all $n \geq N$, and all $s_{1}, \ldots, s_{n} \in G$ (not necessarily distinct), there is some $\mu \in M(G)^{+}$so that

$$
\left\|s_{1} * \mu \vee \ldots \vee s_{n} * \mu\right\|<\varepsilon n\|\mu\|
$$

Proof. We will show this by showing that condition (M) is equivalent to (L) We choose $\varepsilon \in(0,1)$ and fix $n \in \mathbb{N}$ as required in both cases.

Firstly, we know by Theorem 2.5.3 it is possible to embed $L^{1}(G)$ into $M(G)$. In particular this embedding preserves norm, translations and joins. We can show that this is enough to give us eq. 4.8) from condition (L) So take $f \in L^{1}(G)^{+}$satisfying(L) for the given $\varepsilon$ and $n$. Recall that we define $\mu_{f}(A)=\int_{A} f \mathrm{~d} \lambda$. We then have that

$$
\begin{aligned}
\left\|s_{1} * \mu_{f} \vee \ldots \vee s_{n} * \mu_{f}\right\| & =\left\|\mu_{s_{1} * f \vee \cdots \vee s_{n} * f}\right\| \\
& =\left\|s_{1} * f \vee \ldots \vee s_{n} * f\right\| \\
& <\varepsilon n\|f\| \\
& =\varepsilon n\left\|\mu_{f}\right\|
\end{aligned}
$$

Thus $\mu_{f}$ satisfies condition (M),

In the other direction, take $\mu \in M(G)^{+}$so that $\mu$ satisfies (M) (again for the given $\varepsilon$ and $n$ ). Now take any $h \in L^{1}(G)^{+}$, and define $f \in L^{1}(G)^{+}$by $f:=\mu * h$. We note that we have $\|f\|=\|\mu\|\|h\|$, which holds as both $\mu$ and $h$ are positive. Then it follows that

$$
\begin{aligned}
\left\|s_{1} * f \vee \ldots \vee s_{n} * f\right\| & =\left\|s_{1} * \mu * h \vee \ldots \vee s_{n} * \mu * h\right\| \\
& \leq\left\|\left(s_{1} * \mu \vee \ldots \vee s_{n} * \mu\right) * h\right\| \\
& =\left\|s_{1} * \mu \vee \ldots \vee s_{n} * \mu\right\|\|h\| \\
& <\varepsilon n\|\mu\|\|h\| \\
& =\varepsilon n\|f\|
\end{aligned}
$$

Hence $f$ satisfies condition (L) 


\subsection{Subgroups \& Homomorphisms}

With these new characterisations we can show some interesting properties about pseudo-amenable groups. In particular we will show that certain operations (such as taking subgroups, or images of homomorphisms) will preserve pseudo-amenability.

We start with the case of subgroups and state the following definition.

Definition 4.3.1. Let $G$ be a locally compact group, and $H$ a closed subgroup. A Bruhat function for $H$ is a function $\beta: G \rightarrow \mathbb{R}$ satisfying

- For every compact $C \subseteq G$, there is a continuous function $\psi \geq 0$ so that $\left.\psi\right|_{C H}=\left.\beta\right|_{C H}$.

- For every $x \in G$ we have

$$
\int_{H} \beta(x y) \mathrm{d}_{H} y=1
$$

We first note that for every locally compact group $G$ and closed subgroup $H$, there exists a Bruhat function for $H$. This is shown in Chapter 8 of Reiter [20].

We can now show that a closed subgroup of pseudo-amenable group is itself pseudo-amenable. For this, we adopt a proof to that given in Paterson [18, Section (1.11)], where a similar statement is proved for amenable groups. However, the case for pseudo-amenable groups is more involved as we cannot simply take a left-invariant mean.

Theorem 4.3.2. Let $G$ be a locally compact pseudo-amenable group. Let $H$ be a closed subgroup of $G$. Then $H$ itself is pseudo-amenable.

Proof. We let $\varepsilon>0$ and take any $n$ large enough and any $s_{1}, \ldots, s_{n} \in H$ and some $f \in L^{1}(G)^{+}$satisfying (L). In fact we can assume by Lemma 4.2.3 that $f \in C_{c}(G)^{+}$. 
Let $\beta$ be a Bruhat function for $H$. We define

$$
g(y):=\int_{G} f(x) \beta\left(x^{-1} y\right) \mathrm{d}_{G} x
$$

We can find some continuous function $\psi \geq 0$ so that $\left.\psi\right|_{C^{-1} H}=\left.\beta\right|_{C^{-1} H}$ where $C$ is the support of $f$. This then gives us that

$$
g(y)=\int_{G} f(x) \psi\left(x^{-1} y\right) \mathrm{d}_{G} x
$$

As both $f$ and $\psi$ are continuous, we can now see that $g$ must be measurable. Next we show $g \in L^{1}(H)^{+}$. Note that $g \geq 0$ as both $f \geq 0$ and $\beta \geq 0$. We can see that

$$
\begin{aligned}
\int_{H} g(y) \mathrm{d}_{H} y & =\int_{H} \int_{G} f(x) \beta\left(x^{-1} y\right) \mathrm{d}_{G} x \mathrm{~d}_{H} y \\
& =\int_{G} f(x) \int_{H} \beta\left(x^{-1} y\right) \mathrm{d}_{H} y \mathrm{~d}_{G} x \\
& =\int_{G} f(x) \mathrm{d}_{G} x
\end{aligned}
$$

so it follows that $\|g\|_{L^{1}(H)}=\|f\|_{L^{1}(G)}$.

Now if we consider a translation of $g$ by $s \in H$, it is not difficult to see that

$$
(s * g)(y)=\int_{G}(s * f)(x) \beta\left(x^{-1} y\right) \mathrm{d}_{G} x
$$

From this we can show that $\left\|s_{1} * g \vee \ldots \vee s_{n} * g\right\| \leq\left\|s_{1} * f \vee \ldots \vee s_{n} * f\right\|$. For simplicity we argue the case where $n=2$, but the argument may be easily 
generalised for any $n$.

$$
\begin{aligned}
& \left\|s_{1} * g \vee s_{2} * g\right\| \\
= & \int_{H}\left(s_{1} * g \vee s_{2} * g\right)(y) \mathrm{d}_{H} y \\
= & \int_{H}\left[\int_{G}\left(s_{1} * f\right)(x) \beta\left(x^{-1} y\right) \mathrm{d}_{G} x\right] \vee\left[\int_{G}\left(s_{2} * f\right)(x) \beta\left(x^{-1} y\right) \mathrm{d}_{G} x\right] \mathrm{d}_{H} y \\
\leq & \left.\int_{H} \int_{G}\left[\left(s_{1} * f\right)(x) \beta\left(x^{-1} y\right)\right] \vee\left[\left(s_{1} * g\right)(x) \beta\left(x^{-1} y\right)\right)\right] \mathrm{d}_{G} x \mathrm{~d}_{H} y \\
= & \int_{H} \int_{G}\left(s_{1} * f \vee s_{2} * f\right)(x) \beta\left(x^{-1} y\right) \mathrm{d}_{G} x \mathrm{~d}_{H} y \\
= & \int_{G}\left(s_{1} * f \vee s_{2} * f\right)(x) \int_{H} \beta\left(x^{-1} y\right) \mathrm{d}_{H} y \mathrm{~d}_{G} x \\
= & \int_{G}\left(s_{1} * f \vee s_{2} * f\right)(x) \mathrm{d}_{G} x \\
= & \left\|s_{1} * f \vee s_{2} * f\right\|
\end{aligned}
$$

Thus it follows that $g$ satisfies (L), as

$$
\left\|s_{1} * g \vee \ldots \vee s_{n} * g\right\| \leq\left\|s_{1} * f \vee \ldots \vee s_{n} * f\right\|<\varepsilon n\|f\|=\varepsilon n\|g\|
$$

So $H$ is pseudo-amenable.

Our next theorem will show that the image of a pseudo-amenable group under a homomorphism is also pseudo-amenable. We shall use condition (M) to show this. The proof will be a two step process. First we use the measure as given by (M) and our homomorphism to construct an element of the dual $C_{0}(H)$. With this we use the Riesz-Markov theorem to create the desired measure on $H$, and we show it satisfies the necessary properties for pseudo-amenability.

Theorem 4.3.3. Let $G$ and $H$ be locally compact groups, and let $\pi: G \rightarrow H$ be a surjective homomorphism. If $G$ is pseudo-amenable, then so is $H$.

Proof. We shall use condition (M) from Theorem 4.2.5 to prove this, using a linear mapping from $M(G)$ to $M(H)$. We define this mapping as follows. 
First let $\mu \in M(G)$. Let $\varphi_{\mu} \in C_{0}(H)^{*}$ be defined as

$$
\varphi_{\mu}(h):=\int_{G} h \circ \pi \mathrm{d} \mu
$$

It is easy to see that this mapping is linear. Moreover we claim that $\left\|\varphi_{\mu}\right\| \leq$ $\|\mu\|$, so that this operator is bounded. We can see this as

$$
\left|\varphi_{\mu}(h)\right|=\left|\int_{G} h \circ \pi \mathrm{d} \mu\right| \leq \int_{G}\|h \circ \pi\|_{\infty} \mathrm{d}|\mu| \leq\|h\|_{\infty}\|\mu\|
$$

In the case where $\mu \geq 0$, we claim that $\left\|\varphi_{\mu}\right\|=\|\mu\|$. To show this we need to construct a sequence of positive elements $h_{n} \in C_{0}(H)$ with the property that

$$
\lim _{n \rightarrow \infty} \frac{\varphi_{\mu}\left(h_{n}\right)}{\left\|h_{n}\right\|} \geq\|\mu\|
$$

We construct these $h_{n}$ as follows. First we know that $\mu$ is regular. So there must be some sequence of compact sets $K_{n}$ so that $\lim _{n \rightarrow \infty} \mu\left(K_{n}\right)=\mu(G)$. Let $L_{n}=\pi\left(K_{n}\right)$, which by continuity are also compact. Now, we can use Proposition 2.1.12 to find some $h_{n} \in C_{c}(H) \subseteq C_{0}(H)$ with $\left\|h_{n}\right\|=1$ so that $h_{n}(x)=1$ for all $x \in L_{n}$.

Now take $\varepsilon>0$, and choose $n$ so that $\mu\left(K_{n}\right) \geq \mu(G)-\varepsilon$. Then we have that

$$
\varphi_{\mu}\left(h_{n}\right)=\int_{G} h_{n} \circ \pi \mathrm{d} \mu \geq \int_{K_{n}} h_{n} \circ \pi \mathrm{d} \mu=\int_{K_{n}} 1 \mathrm{~d} \mu=\mu\left(K_{n}\right) \geq \mu(G)-\varepsilon
$$

Noting that $\left\|h_{n}\right\|=1$, we can take the limit as $\varepsilon \rightarrow 0$, so that these $h_{n}$ satisfy eq. 4.14. This proves our claim that $\|\mu\|=\left\|\varphi_{\mu}\right\|$.

Now, by the Riesz-Markov Representation theorem, there is some measure $\mu_{\pi} \in M(H)$ so that

$$
\int_{H} h \mathrm{~d} \mu_{\pi}=\varphi_{\mu}(h)=\int_{G} h \circ \pi \mathrm{d} \mu
$$

for every $h \in C_{0}(H)$. We note that if $\mu$ is positive, then so is $\mu_{\pi}$, and furthermore we have $\left\|\mu_{\pi}\right\|=\left\|\varphi_{\mu}\right\|=\|\mu\|$. 
We will show that the mapping $\mu \mapsto \mu_{\pi}$ is sufficiently nice so that if we take $\mu \in M(G)^{+}$that satisfies (M), then so will $\mu_{\pi} \in M(H)^{+}$for the same values of $\varepsilon$ and $n$.

Now consider a translation of $\mu_{\pi}$. In particular, for any $s \in G$ and positive $h \in C_{0}(H)$, we have that

$$
\begin{aligned}
\left\langle\pi(s) * \mu_{\pi}, h\right\rangle & =\iint h(x y) \mathrm{d} \delta_{\pi(s)}(x) \mathrm{d} \mu_{\pi}(y) \\
& =\int h(\pi(s) y) \mathrm{d} \mu_{\pi}(y) \\
& =\int h(\pi(s) \pi(y)) \mathrm{d} \mu(y) \\
& =\int h(\pi(s y)) \mathrm{d} \mu(y) \\
& =\int h \circ \pi \mathrm{d} s * \mu \\
& =\left\langle(s * \mu)_{\pi}, h\right\rangle
\end{aligned}
$$

Since this holds for all $h \in C_{0}(H)$, then we have that $\pi(s) * \mu_{\pi}=(s * \mu)_{\pi}$.

Next, we take $\mu \in M(G)^{+}$. Then for any positive $h \in C_{0}(H)$, we have

$$
\begin{aligned}
\left\langle\left(\mu_{\pi}\right)^{+}, h\right\rangle & =\sup \left\{\left\langle\mu_{\pi}, f\right\rangle: f \in C_{0}(H), 0 \leq f \leq h\right\} \\
& =\sup \left\{\langle\mu, f \circ \pi\rangle: f \in C_{0}(H), 0 \leq f \leq h\right\} \\
& \leq \sup \left\{\langle\mu, g\rangle: g \in C_{0}(G), 0 \leq g \leq h \circ \pi\right\} \\
& =\left\langle\mu^{+}, h \circ \pi\right\rangle \\
& =\left\langle\left(\mu^{+}\right)_{\pi}, h\right\rangle
\end{aligned}
$$

From this it follows that for any $\mu, \nu \in M(G)^{+}$, we have that $\left(\mu_{\pi} \vee \nu_{\pi}\right)^{+} \leq$ $(\mu \vee \nu)_{\pi}^{+}$.

With this, we can take any $\varepsilon \in(0,1)$, and let $n$ be sufficiently large. Take any $t_{1}, \ldots, t_{n} \in H$, and since $\pi$ is surjective, we can find $s_{1}, \ldots, s_{n} \in G$ so that $\pi\left(s_{i}\right)=t_{i}$. Now choose $\mu \in M(G)$ satisfying condition (M) for 
$s_{1}, \ldots, s_{n}$. Then, it follows that

$$
\begin{aligned}
\left\|t_{1} * \mu_{\pi} \vee \ldots \vee t_{n} * \mu_{\pi}\right\| & =\left\|\left(s_{1} * \mu\right)_{\pi} \vee \ldots \vee\left(s_{n} * \mu\right)_{\pi}\right\| \\
& \leq\left\|\left(s_{1} * \mu \vee \ldots \vee s_{n} * \mu\right)_{\pi}\right\| \\
& =\left\|s_{1} * \mu \vee \ldots \vee s_{n} * \mu\right\| \\
& <\varepsilon n\|\mu\| \\
& =\varepsilon n\left\|\mu_{\pi}\right\|
\end{aligned}
$$

Hence $\mu_{\pi}$ satisfies (M), and so $H$ is pseudo-amenable.

Corollary 4.3.4. Let $G$ be a pseudo-amenable group, and $N$ a closed subgroup. Then the quotient group $G / N$ is pseudo-amenable.

Proof. We know that the canonical mapping $q: G \rightarrow G / N$ where we have $q(x)=x N$ is a surjective homomorphism. By Theorem 4.3.3, it follows that $G / N$ is pseudo-amenable.

We now wish to see if we can reverse this process. In particular we want to show the pseudo-amenability of a group by looking at its subgroups and quotient groups. Before we attempt this, we need some method of relating the Haar measure of our group $G$ and the groups $H$ and $G / H$. Some details of this are covered by Folland in [6, Pages 56-57]. Most importantly is the result stated in Theorem 2.49. In essence, if we have a closed normal subgroup $H$, then we can normalise the Haar measures in each of $G, H$, and $G / H$, so that for any function $f \in C_{c}(G)$ we have

$$
\int_{G} f(x) \mathrm{d} x=\int_{G / H} P f(x H) \mathrm{d}(x H)
$$

where we define $\operatorname{Pf} \in C_{c}(G / H)$ by

$$
P f(x H):=\int_{H} f(x h) \mathrm{d} h
$$

We need a slightly altered version of this statement in terms of characteristic functions, which we prove as follows. 
Lemma 4.3.5. Let $G$ be a locally compact group, and $H$ a closed normal subgroup. For any compact set $K$, we have

$$
\lambda(K)=\int_{G / H} \int_{H} \chi_{K}(x h) \mathrm{d} h \mathrm{~d}(x H)
$$

Proof. Firstly we shall for convenience define

$$
\varphi(f):=\int_{G / H} \int_{H} f(x h) \mathrm{d} h \mathrm{~d}(x H)
$$

for any function $f$ for which the right hand side is defined. Our aim then is to show that $\lambda(K)=\varphi\left(\chi_{K}\right)$ for all compact sets $K$.

To prove this result we shall need to use Proposition 7.4.4 in Cohn [1]. This tells us that if $f: G \rightarrow \mathbb{R}$ is a positive lower semicontinuous function, and if $\mathcal{K}$ is a family of positive lower semitcontinuous functions such that

$$
f(x)=\sup \{g(x): g \in \mathcal{K}\}
$$

then

$$
\int_{G} f(x) \mathrm{d} x=\sup \left\{\int_{G} g(x) \mathrm{d} x: g \in \mathcal{K}\right\}
$$

Here we say a function is lower semicontinuous if for every $\alpha \in \mathbb{R}$, we have that the set $\{x \in G: f(x)>\alpha\}$ is open. One may easily verify that $\chi_{U}$ for any open set $U$ is lower semicontinuous.

Now, for any open set $U$, we may use Proposition 2.1.12 to show that $\chi_{U}(x)=\sup \left\{f(x): f \in C_{c}(G), 0 \leq f \leq \chi_{U}\right\}$. Using Proposition 7.4.4 from Cohn, we then have that

$$
\begin{aligned}
\int_{H} \chi_{U}(x h) \mathrm{d} h & =\sup \left\{\int_{H} f(x h) \mathrm{d} x: f \in C_{c}(G), 0 \leq f \leq \chi_{U}\right\} \\
& =\sup \left\{\operatorname{Pf}(x H): f \in C_{c}(G), 0 \leq f \leq \chi_{U}\right\}
\end{aligned}
$$

We note here that the function $P f$ is continuous, so we can apply Proposition 7.4.4 to this expression as well. In fact, we shall need to apply it two 
more times, and in doing so we obtain

$$
\begin{aligned}
\varphi\left(\chi_{U}\right) & =\int_{G / H} \int_{H} \chi_{U}(x h) \mathrm{d} h \mathrm{~d}(x H) \\
& =\sup \left\{\int_{G / H} \operatorname{Pf}(x H) \mathrm{d}(x H): f \in C_{c}(G), 0 \leq f \leq \chi_{U}\right\} \\
& =\sup \left\{\int_{G} f(x) \mathrm{d} x: f \in C_{c}(G), 0 \leq f \leq \chi_{U}\right\} \\
& =\int_{G} \sup \left\{f(x): f \in C_{c}(G), 0 \leq f \leq \chi_{U}\right\} \mathrm{d} x \\
& =\int_{G} \chi_{U}(x) \mathrm{d} x=\lambda(U)
\end{aligned}
$$

This proves the equality we desire for $\lambda$-finite open sets. In the case where we have a compact set $K$, we can find a $\lambda$-finite open set $U$ so that $K \subseteq U$. It then follows that both $U$ and $U \backslash K$ are $\lambda$-finite open sets, so that we have $\varphi\left(\chi_{U}\right)=\lambda(U)$ and $\varphi\left(\chi_{K \backslash U}\right)=\lambda(K \backslash U)$. From this we finally get that

$$
\lambda(K)=\lambda(U)-\lambda(U \backslash K)=\varphi\left(\chi_{U}\right)-\varphi\left(\chi_{U \backslash K}\right)=\varphi\left(\chi_{U}-\chi_{U \backslash K}\right)=\varphi\left(\chi_{K}\right)
$$

thus proving our result.

In the ideal case, we would only need a closed normal subgroup $H$ and the corresponding quotient group $G / H$ to be pseudo-amenable in order to induce pseudo-amenability in $G$. However, we do in fact require our subgroup $H$ to be amenable.

Theorem 4.3.6. Let $G$ be a locally compact group, and $H$ a closed normal subgroup. If $H$ is amenable and $G / H$ is pseudo-amenable, then $G$ is pseudo-amenable.

Proof. Firstly we let $\pi: G \rightarrow G / H$ denote the canonical mapping where $\pi(x)=x H$. We shall denote the Haar measures on $G, H$ and $G / H$ by $\lambda_{G}$, $\lambda_{H}$ and $\lambda_{G / H}$ respectively.

Now let $\varepsilon>0$, and choose $N$ as required for the pseudo-amenability of $G / H$. Take any finite set $F \subseteq G$ so that $|F| \geq N$. Now it is possible that $|\pi(F)|<N$, so we cannot directly apply pseudo-amenability here. 
However, in this case we may simply take any finite set $X$ so that $\pi(F) \subseteq$ $X$ and $|X|=|F| \geq N$. This gives us that there is some compact set $C^{\prime} \subseteq$ $G / H$ so that

$$
\lambda_{G / H}\left(\pi(F) C^{\prime}\right) \leq \lambda_{G / H}\left(X C^{\prime}\right)<\frac{\varepsilon}{2}|F| \lambda_{G / H}\left(C^{\prime}\right)
$$

We note that the right side of this equation is $|F|$ instead of $|\pi(F)|$.

Now, for any compact set $C^{\prime}$ in $G / H$, we can find a compact set $C$ in $G$ so that $\pi(C)=C^{\prime}$ (the proof of this is given by Folland in [6, Lemma 2.46]). So this means we can find a compact set $C \subseteq G$ so that

$$
\lambda_{G / H}(\pi(F C))<\frac{\varepsilon}{2}|F| \lambda_{G / H}(\pi(C))
$$

Next we define

$$
K=(F C)^{-1} F C \cap H
$$

It is clear from this definition that $K \subseteq H$. Moreover, we know that $F C$ is compact, and this gives us that $K$ must also be compact. We can use the amenability of $H$ via eq. 4.1) to find some compact set $L \subseteq H$ so that

$$
\lambda_{H}(K L)<2 \lambda_{H}(L)
$$

Now, for any $x \in G$ and $h \in H$ where $x h \in F C L$, we clearly have that $x H \in \pi(F C)$. Using Lemma 4.3 .5 gives us that

$$
\begin{aligned}
\lambda_{G}(F C L) & =\int_{G / H} \int_{H} \chi_{F C L}(x h) \mathrm{d} h \mathrm{~d}(x H) \\
& =\int_{\pi(F C)} \int_{H} \chi_{F C L}(x h) \mathrm{d} h \mathrm{~d}(x H) \\
& =\int_{\pi(F C)} \lambda_{H}\left(x^{-1} F C L \cap H\right) \mathrm{d}(x H) \\
& =\int_{\pi(F C)} \lambda_{H}\left(\left(x^{-1} F C \cap H\right) L\right) \mathrm{d}(x H) \\
& \leq \int_{\pi(F C)} \lambda_{H}(K L) \mathrm{d}(x H) \\
& =\lambda_{G / H}(\pi(F C)) \lambda_{H}(K L)
\end{aligned}
$$


Next, for any $x \in C$ and $h \in H$, we have that if $h \in L$ then $x h \in C L$. Thus it follows that $\chi_{L}(h) \leq \chi_{C L}(x h)$. From this we obtain

$$
\begin{aligned}
\lambda_{G}(C L) & =\int_{G / H} \int_{H} \chi_{C L}(x h) \mathrm{d} h \mathrm{~d}(x H) \\
& =\int_{\pi(C)} \int_{H} \chi_{C L}(x h) \mathrm{d} h \mathrm{~d}(x H) \\
& \geq \int_{\pi(C)} \int_{H} \chi_{L}(h) \mathrm{d} h \mathrm{~d}(x H) \\
& =\lambda_{G / H}(\pi(C)) \lambda_{H}(L)
\end{aligned}
$$

We can finally use these results to get that

$$
\begin{aligned}
\lambda_{G}(F C L) & \leq \lambda_{G / H}(\pi(F C)) \lambda_{H}(K L) \\
& <\varepsilon|F| \lambda_{G / H}(\pi(C)) \lambda_{H}(L) \\
& \leq \varepsilon|F| \lambda_{G}(C L)
\end{aligned}
$$

which is precisely what we need to show the pseudo-amenability of $G$.

A natural corollary of this theorem is that we can construct pseudoamenable groups using group products as follows.

Corollary 4.3.7. If $G$ is an amenable group and $H$ a pseudo-amenable group, then $G \times H$ is pseudo-amenable.

Proof. We know that $G \times\{e\}$ is a closed normal subgroup of $G \times H$, whose corresponding quotient group is isomorphic to $H$. By Theorem 4.3.6, we get that $G \times H$ is pseudo-amenable.

\subsection{Similarities to Amenability}

In the previous section, we showed some properties of pseudo-amenable groups that closely mimic the behaviour of amenable groups. Unfortunately however, we did not show all the results equivalent to those presented in Theorems 3.2.3 to 3.2.6. 
The first instance of this is constructing a group from its subgroup and quotient group. In particular we know that if a normal subgroup $H$ and its quotient group $G / H$ are both amenable, then $G$ is amenable as well (see Theorem 3.2.5). The case for pseudo-amenability is similar, however we have in Theorem 4.3 .6 that our subgroup $H$ is also required to be amenable.

We also showed that a group is pseudo-amenable if all its densely countably generated subgroups are. If one can generalise this statement to only require (densely) finitely generated subgroups, then this would be equivalent to showing that a direct union of pseudo-amenable groups is pseudo-amenable.

One could argue that the underlying issue in both these cases is that we do not have a bound for $N$ in our condition. If we for example consider condition (SF), which is equivalent to amenability, it tells us that for any $\varepsilon>0$ and finite set $F$, we can find a compact set $C$ so that we have $\lambda(F C)<(\varepsilon+1) \lambda(C)$. So, for any amenable group, we can always satisfy (PA) by simply choosing $N \geq(\varepsilon+1) / \varepsilon$. The existence of such a bound would allow us to resolve the issues discussed previously. An alternative solution would be to show that pseudo-amenability and amenability are equivalent.

It is also possible that these are not equivalent, and perhaps the simplest way to show this would be by constructing a counterexample. However, as the following theorem tells us, such a group would not contain $F_{2}$ as a subgroup. We use an outline of the proof presented by Pham [19. Proposition 5.9].

Proposition 4.4.1. Let $G$ be a locally compact group that contains $F_{2}$. Then $G$ is not pseudo-amenable.

Proof. Firstly, it is known that $F_{2}$ contains the countably generated group $F_{\mathbb{N}}$ as a subgroup, so we will without loss of generality assume that we have $G=F_{\mathbb{N}}$. For any $n \in \mathbb{N}$ we will define $\hat{F}_{n}=\left\{s_{1}, \ldots, s_{n}\right\}$, where these 
are the first $n$ basis elements of $F_{\mathbb{N}}$. We claim that for any finite (compact) subset $C \subseteq F_{\mathbb{N}}$, we have that

$$
\left|\hat{F}_{n} C\right|>(n-1)|C|
$$

We will prove this claim inductively, by inducting on $C$. To do so, we let $x_{1}$ be any element in $C$ with minimal length. We define $C_{1}=\left\{x_{1}\right\}$, and we easily see that

$$
\left|\hat{F}_{n} C_{1}\right|>(n-1)\left|C_{1}\right|
$$

We define $C_{k}$ inductively by adding any element of minimal length at each step. That is, we take $x_{k} \in C \backslash C_{k-1}$ so that $\ell(x) \leq \ell(z)$ for every $z \in C \backslash C_{k-1}$, where $\ell$ is the length function on the free group (so $\ell(x)$ is the number of characters in $x$ in its reduced form). We then define $C_{k}=C_{k-1} \cup\left\{x_{k}\right\}$.

For now, we shall assume that the set $\hat{F}_{n} C_{k-1} \cap \hat{F}_{n} x$ contains at most one element, and we will prove this fact later. With this we show the inductive step as

$$
\begin{aligned}
\left|\hat{F}_{n} C_{k}\right| & =\left|\hat{F}_{n} C_{k-1} \cup \hat{F}_{n} x_{k}\right| \\
& \geq\left|\hat{F}_{n} C_{k-1}\right|+\left|\hat{F}_{n} x_{k}\right|-1 \\
& >(n-1)\left|C_{k-1}\right|+n-1 \\
& =(n-1)\left|C_{k}\right|
\end{aligned}
$$

So to complete the proof we only need to show that $\left|\hat{F}_{n} C_{k-1} \cap \hat{F}_{n} x\right| \leq 1$. So let $y$ be an element of this set, so we can write $y=s_{i} c=s_{j} x$ for some $s_{i}, s_{j} \in \hat{F}_{n}$ and $c \in C_{k-1}$. We can show that $y$ must necessarily be unique. To see this, we first note that the length of $c$ is at most the length of $x$. Now suppose that the first character of $x$ (in its reduced form) is $s_{m}$ for $s_{m}$ in the basis of $\hat{F}_{n}$. Then since $\ell(c) \leq \ell(x)$, it follows that we must have that $s_{i}=s_{j}$ and therefore $c=x$, which is a contradiction. Hence we must have that $x$ starts with $s_{m}^{-1}$, and moreover we must have $s_{j}=s_{m}$. This is enough to uniquely determine $y$, thus proving our claim.

So, if we were to show that pseudo-amenability and amenability were two distinct classes of groups by using an example, then such an example 
would necessarily be a nonamenable group that does not contain $F_{2}$. The existence of such groups was a long standing problem, commonly referred to as Von Neumann Conjecture, and was resolved by Ol'shanskii in 1979 [17] by constructing an explicit (though very complex) example of such a group. Recently, simpler counterexamples have been found by Monod [15] and by Lodha and Moore [12], so these may prove to be easier to analyse. It is not known if these groups are pseudo-amenable or not. 


\section{Bibliography}

[1] CoHn, D. Measure Theory. Birkhäuser Boston, 1994.

[2] Dales, H. G., and Polyakov, M. E. Homological properties of modules over group algebras. Proceedings of the London Mathematical Society 89, 2 (2004), 390-426.

[3] DAY, M. M. Amenable semigroups. Illinois J. Math. 1, 4 (12 1957), 509-544.

[4] Dougherty, R., And Foreman, M. Banach-Tarski decompositions using sets with the property of Baire. J. Amer. Math. Soc. 7, 1 (1994), $75-124$.

[5] Emerson, W. R., AND Greenleaf, F. P. Covering properties and Følner conditions for locally compact groups. Mathematische Zeitschrift 102, 5 (Oct 1967), 370-384.

[6] Folland, G. A Course in Abstract Harmonic Analysis. Studies in Advanced Mathematics. Taylor \& Francis, 1994.

[7] FøLNER, E. On groups with full Banach mean value. MATHEMATICA SCANDINAVICA 3 (Dec. 1955), 243-254.

[8] GReEnleaf, F. P. Invariant Means on Topological Groups (Mathematics Studies). Van Nostrand Reinhold Inc.,U.S., 1969. 
[9] HAAR, A. Der massbegriff in der theorie der kontinuierlichen gruppen. Annals of Mathematics 34, 1 (1933), 147-169.

[10] (HTTPS: / / MATH.STACKEXCHANGE.COM/USERS/83702 / DANIEL FISCHER), D. F. Continuity of modular function. Mathematics Stack Exchange. URL:https://math.stackexchange.com/q/669029 (version: 2014-02-09).

[11] HulAniCKI, A. Means and Følner condition on locally compact groups. Studia Mathematica 27, 2 (1966), 87-104.

[12] Lodha, Y., AND MoOre, J. T. A nonamenable finitely presented group of piecewise projective homeomorphisms. Groups, Geometry, and Dynamics 10, 1 (2016), 177-200.

[13] Loomis, L. An Introduction to Abstract Harmonic Analysis. Higher Mathematics. Van Nostrand, 1953.

[14] LoOmis, L. H. Haar measure in uniform structures. Duke Mathematical Journal 16, 2 (jun 1949), 193-208.

[15] MONOD, N. Groups of piecewise projective homeomorphisms. Proceedings of the National Academy of Sciences 110, 12 (2013), 4524-4527.

[16] NAMIOKA, I. Folner's conditions for amenable semi-groups. MATHEMATICA SCANDINAVICA 15 (Jun. 1964), 18-28.

[17] OL'SHANSKII, A. J. An infinite simple torsion-free Noetherian group. Izv. Akad. Nauk SSSR Ser. Mat. 43, 6 (1979), 1328-1393.

[18] PAterson, A. Amenability. Mathematical surveys and monographs. American Mathematical Soc., 1988.

[19] PHAM, H. L. A combinatorial characterisation of amenable locally compact groups. Journal of the London Mathematical Society 98, 3 (2018), 638-660. 
[20] Reiter, H. Classical Harmonic Analysis and Locally Compact Groups. Oxford Mathematical Monographs. Clarendon P., 1968.

[21] ReITER, H. J. Investigations in harmonic analysis. Transactions of the American Mathematical Society 73, 3 (mar 1952), 401-401.

[22] Wagon, S. The Banach-Tarski Paradox. Encyclopedia of Mathematics and its Applications. Cambridge University Press, 1993. 